Bull. Chem. Soc. Ethiop. 2014, 28(3), 423-440.

Printed in Ethiopia

DOI: http://dx.doi.org/10.4314/bcse.v28i3.12

ISSN 1011-3924

(c) 2014 Chemical Society of Ethiopia

\title{
NEW SPIRO (THIO) BARBITURATES BASED ON CYCLOHEXANONE AND BICYCLO [3.1.1]HEPTAN-6-ONE BY NONCONCERTED [1+5] CYCLOADDITION REACTION AND THEIR CONFORMATIONAL STRUCTURES
}

\author{
Nader Noroozi Pesyan ${ }^{1 *}$, Sirous Noori $^{1}$, Soodabeh Poorhassan ${ }^{1}$ and Ertan Şahin ${ }^{2}$ \\ ${ }^{1}$ Faculty of Chemistry, Urmia University, 57159, Urmia, Iran \\ ${ }^{2}$ Department of Chemistry, Faculty of Science, Atatürk University, 25240 Erzurum, Turkey
}

(Received July 22, 2013; revised May 7, 2014)

\begin{abstract}
Crossed-aldol condensation reaction of aromatic aldehydes with ketones such as; acetone and cyclohexanone leads to the efficient formation of cross conjugated $\alpha, \beta$-unsaturated ketones in excellent yield. The intermolecular and then intramolecular Michael addition reaction of $\alpha, \beta$-unsaturated ketones derived from acetone and cyclohexanone with (thio)barbituric acids lead to synthesis new type of 7,11-diaryl-2,4diazaspiro[5.5] undecane-1,3,5,9-tetraone and 2,4-diaryl-1' $H$-spiro[bicyclo[3.3.1]nonane-3,5'-pyrimidine]$2^{\prime}, 4^{\prime}, 66^{\prime}, 9\left(3^{\prime} H\right)$-tetraone, respectively in good yield. Structure elucidation is carried out by ${ }^{1} \mathrm{H}$ NMR, ${ }^{13} \mathrm{C}$ NMR, FTIR, UV-Visible, mass spectroscopy and X-ray crystallography techniques. A possible mechanism of the formation is discussed. The structural conformation also demonstrated by coupling constants derived from dihedral angles between vicinal and geminal protons. The ${ }^{1} \mathrm{H}$ NMR spectra of $\mathrm{NH}$ protons of spiro compounds derived from barbituric acid show a broad singlet peak instead, these protons in the spiro compounds derived from thiobarbituric acid show two distinct peaks.
\end{abstract}

KEY WORDS: Crossed-aldol condensation, Michael addition, [1+5] Cycloaddition, Barbituric acid, Conformation, Spiro barbiturate

\section{INTRODUCTION}

The crossed-aldol condensation reaction is an efficient route for the synthesis of $\alpha, \alpha^{\prime}-$ bis(substituted benzylidene)cycloalkanones as precursors for the synthesis of biological pyrimidine derivatives [1-4] These compounds have gained a lot of attention due to their uses as agrochemical, pharmaceutical and perfume intermediates [5-8] and as liquid crystal polymer units [9]. Among these reactions, the aldol condensation reaction is useful for the formation of carbon-carbon bonds in many kinds of carbonyl compounds [10-12]. Crossed-aldol condensation can be operated with the aid of strong acids or bases [2, 13]. Several catalytic methods have been achieved for crossed-aldol condensation [4, 14-31].

Several spiro-(thio)barbituric acid derivatives in which the active methylene carbon of (thio)barbituric acid is substituted by an unsubstituted cyclobutane or cyclohexane ring [32, 33] have been synthesized, but no pharmacological data is obtained with these compounds. Spiro compounds have been reported to possess biological activities that include anti-inflammatory activity [34], anticonvulsant, CNS depressant [35], narcotic [36], hypotensive, analgesic [37]. Several works have been reported for the synthesis of other spiro barbiturates [38-44].

As we searched in the literature, there are some reports about spiro barbiturates based on bis-arylideneacetones [36, 45] and no report about spiro barbiturates based on 2,6-bisarylidenecyclohexanones. Based on these concepts, in this research, we report a new and facile route for the synthesis of 5,5-two substituted spiro-barbiturates by the condensation reaction of $\mathrm{N}$-substituted and unsubstituted (thio)barbituric acids with dibenzalacetone and 2,6dibenzylidenecyclohexanone derivatives.

\section{EXPERIMENTAL}

General

The drawing and nomenclature of compounds were done by ChemBioDraw Ultra 8.0 version software. Melting points were measured with a digital melting point apparatus (Electrothermal)

*Corresponding author. E-mail: n.noroozi@urmia.ac.ir ; nnp403@gmail.com 
and were uncorrected. IR spectra were determined in the region $4000-400 \mathrm{~cm}^{-1}$ on a NEXUS 670 FT IR spectrometer by preparing $\mathrm{KBr}$ pellets. The ${ }^{1} \mathrm{H}$ and ${ }^{13} \mathrm{C}$ NMR spectra were recorded on Bruker 300 FT-NMR at 300 and $75 \mathrm{MHz}$, respectively (Urmia University, Urmia, Iran). ${ }^{1} \mathrm{H}$ and ${ }^{13} \mathrm{C}$ NMR spectra were obtained on solution in DMSO- $d_{6}$ and/or in $\mathrm{CDCl}_{3}$ as solvents using TMS as internal standard. The data are reported as $(\mathrm{s}=$ singlet, $\mathrm{d}=$ doublet, $\mathrm{t}=$ triplet, $\mathrm{q}=$ quartet, $\mathrm{m}=$ multiplet or unresolved, bs = broad singlet, coupling constant(s) in Hz, integration). All reactions were monitored by TLC with silica gel-coated plates (EtOAc:cyclohexane, 8:10, v:v). The mass analysis performed using mass spectrometer (Agilent Technology (HP) type, MS Model: 5973 Network Mass Selective Detector Electron Impact (EI) $70 \mathrm{eV}$ ), ion source temperature was $230{ }^{\circ} \mathrm{C}$ (Tehran University, Tehran, Iran). The electronic spectra were performed by T80 UV-Vis (PG Instruments Ltd) spectrometer (Urmia University, Urmia, Iran). Compounds 3 were synthesized based on reported literature [25]. Compounds 1a-k, 2a', 2c', $\mathbf{4 a} \mathbf{4}-\mathbf{4 d}$ " and used solvents purchased from Merck and Across without further purification.

Synthesis

General procedures for the preparation of $\mathbf{3 a a ^ { \prime }}$ through $\mathbf{3} \mathbf{k a}^{\prime}$ and $\mathbf{3 a b ^ { \prime }}$ through $\mathbf{3} \mathbf{k} \boldsymbol{b}^{\prime}$. In a round bottom flask equipped with an ice-bath, $2.5 \mathrm{~g} \mathrm{NaOH}$ dissolved in $20 \mathrm{~mL}$ distilled water then added acetone $(1.16 \mathrm{~g}, 0.02 \mathrm{~mol})$ and benzaldehyde $(4.24 \mathrm{~g}, 0.04 \mathrm{~mol})$ then added $20 \mathrm{~mL}$ ethanol dropwise into the reaction mixture. The reaction progression was completed after $2 \mathrm{~h}$. The pale yellow precipitate then washed with $10 \mathrm{~mL}$ chilled ethanol and recrystallized in absolute ethanol (yield: $95 \%$, see Table 1).

Table 1. Physical properties of bis-arylideneacetones (3ca'-3ia') and 2,6-bis-arylidenecyclohexanones $\left(3 \mathbf{a b} \mathbf{b}^{\prime}-\mathbf{3 k b}\right)$

\begin{tabular}{|c|c|c|c|}
\hline Entry & $\begin{array}{c}\text { Bis-arylideneacetones } \\
\text { (cyclohexanones) }\end{array}$ & M.p. $\left({ }^{\circ} \mathrm{C}\right)$ & Color \\
\hline 1 & 3ca' & $196-198$ (Lit. 192-193 [50]) & Yellow \\
\hline 2 & 3ea' & $203-205$ (Lit. 211-213 [50]) & Yellow \\
\hline 3 & 3fa' & $104-106$ (Lit. 104-107 [51]) & Pale yellow \\
\hline 4 & 3ga' & $126-129$ (Lit. 121-122 [50]) & Yellow \\
\hline 5 & 3ia' & $140-143$ (Lit. 141-143 [52]) & Yellow \\
\hline 6 & 3ab' & $185-187$ (Lit. 188-190 [53]) & Yellow \\
\hline 7 & 3cb' & $143-146$ (Lit. 147-148 [53]) & Yellow \\
\hline 8 & 3eb' & $198-202$ (Lit. 201-205 [53]) & Yellow \\
\hline 9 & 3fb' & $117-119$ (Lit. 117-118 [53]) & Yellow \\
\hline 10 & 3gb' & 200-202 (Lit. 203-204 [53]) & Red \\
\hline 11 & 3ib' & $150-154[54]$ & \\
\hline
\end{tabular}

General procedures for the preparation of 7aa'a" through $7 \mathbf{k a}^{\prime} \mathbf{d}^{\prime \prime}$ and $7 \boldsymbol{a b} \mathbf{a}^{\prime \prime}$ through $7 \mathbf{k} \boldsymbol{b}^{\prime} \mathbf{d}^{\prime \prime}$. In a $50 \mathrm{~mL}$ round bottom flask equipped with magnetic stirrer the mixture of 1,3dimethylbarbituric acid $(1.3 \mathrm{mmol}, 0.21 \mathrm{~g})$, dibenzalacetone $(1.3 \mathrm{mmol}, 0.30 \mathrm{~g})$ and triethylamine $(2.1 \mathrm{mmol}, 0.22 \mathrm{~g}, 0.3 \mathrm{~mL})$ in $10 \mathrm{~mL}$ methanol and refluxed for $2-3 \mathrm{~h}$. The yellow color disappeared and consequently white crystalline solid precipitated, filtered off, washed with few $\mathrm{mL}$ of cold ethanol then dried $(0.35 \mathrm{~g}, 70 \%)$.

2,4-Dimethyl-7,11-diphenyl-2,4-diazaspiro[5.5] undecane-1,3,5,9-tetraone (7fa'b") [45]. White crystalline solid, m.p. $153-156{ }^{\circ} \mathrm{C}$, IR (KBr, cm $\left.{ }^{-1}\right) 3037$ (ar.), 2952 (aliph.), 2923 (aliph.), 1717 $(\mathrm{C}=\mathrm{O}), 1675(\mathrm{C}=\mathrm{O}), 1450,1422$ (ar. $\mathrm{C}=\mathrm{C}$ stretch), 1382 (Me bend.), 701(mono subst. ph); ${ }^{1} \mathrm{H}$ $\operatorname{NMR}\left(300 \mathrm{MHz}, \mathrm{CDCl}_{3}\right) \delta 2.64\left(\mathrm{dd}, J=14.7 \mathrm{~Hz}, J=4.5 \mathrm{~Hz}, 2 \mathrm{H}\right.$, diastereotopic $\left.-\mathrm{CH}_{2}{ }^{-}\right), 2.88(\mathrm{~s}$, $3 \mathrm{H}, \mathrm{MeN}), 3.03(\mathrm{~s}, 3 \mathrm{H}, \mathrm{MeN}), 3.75\left(\mathrm{t}, J=14.4 \mathrm{~Hz}, 2 \mathrm{H}\right.$, diastereotopic $\left.-\mathrm{CH}_{2^{-}}\right), 4.04(\mathrm{dd}, J=14.3$ $\mathrm{Hz}, J=4.2 \mathrm{~Hz}, 2 \mathrm{H}$, -CH-ph benzylic proton), 7.07-7.28 (m, $10 \mathrm{H}$, ar.); ${ }^{13} \mathrm{C}$ NMR $(75 \mathrm{MHz}$, 
$\left.\mathrm{CDCl}_{3}\right) \delta 27.9\left(\mathrm{NCH}_{3}\right), 28.3\left(\mathrm{NCH}_{3}\right), 42.9\left(\mathrm{C}_{8}\right.$ or $\left.\mathrm{C}_{10}\right), 50.5\left(\mathrm{C}_{7}\right.$ or $\left.\mathrm{C}_{11}\right), 60.9\left(\mathrm{C}_{6}\right), 127.5(\mathrm{C}$-ar. $)$, 128.6 (C-ar.), 128.8 (C-ar.), 137.1 ( $\mathrm{C}_{\text {ipso }}$ ar. $), 169.5\left(\mathrm{C}_{3}\right), 170.6\left(\mathrm{C}_{1}\right.$ or $\left.\mathrm{C}_{5}\right), 176.0\left(\mathrm{C}_{1}\right.$ or $\left.\mathrm{C}_{5}\right)$, $208.2\left(\mathrm{C}_{9}\right)$; MS ( $\left.\mathrm{m} / \mathrm{z}, \%\right), 390\left(\mathrm{M}^{+}, 45\right), 325$ (6), 286 (15), 258 (100, base peak), 235 (12), 173 (10), 146 (45), 129 (20), 104 (55), 77 (25), 51 (10).

7,11-Bis(4-chlorophenyl)-2,4-diazaspiro[5.5] undecane-1,3,5,9-tetraone (7ca'a') [36]. White crystalline solid; m.p. $98-100{ }^{\circ} \mathrm{C}$; IR $\left(\mathrm{KBr}, \mathrm{cm}^{-1}\right) 3387$ (OH and/or NH), 3211 (NH), 3029 (ar.), 2839 (aliph.), 1710 (C=O), 1590, 1491, 1408, 1091, 1014, 837, 756, 524 (C-Cl stretch); ${ }^{1} \mathrm{H}$ NMR $\left(300 \mathrm{MHz}, \mathrm{DMSO}-d_{6}\right) \delta 2.44\left(\mathrm{dd}, J=14.9 \mathrm{~Hz}, J=3.6 \mathrm{~Hz}, 2 \mathrm{H}\right.$, diastereotopic $\left.-\mathrm{CH}_{2^{-}}\right), 3.43$ (t, $J=13.8 \mathrm{~Hz}, 2 \mathrm{H}$, diastereotopic $-\mathrm{CH}_{2^{-}}$, overlapped with DMSO $\left(\mathrm{H}_{2} \mathrm{O}\right)$ peak), 4.01 (dd, $J=$ $13.8 \mathrm{~Hz}, J=3.9 \mathrm{~Hz}, 2 \mathrm{H},-\mathrm{CH}-$ ph benzylic proton), 7.10 (d, $J=8.1 \mathrm{~Hz}, 4 \mathrm{H}$, ar. $-\mathrm{H}), 7.37$ (d, $J=$ $8.1 \mathrm{~Hz}, 4 \mathrm{H}$, ar.-H), 11.0 (extremely bs, $2 \mathrm{H}, 2 \mathrm{NH}) ;{ }^{13} \mathrm{C}$ NMR $\left(75 \mathrm{MHz}, \mathrm{DMSO}-d_{6}\right) \delta 42.8\left(\mathrm{C}_{8}\right.$ or $\left.\mathrm{C}_{10}\right), 48.4\left(\mathrm{C}_{7}\right.$ or $\left.\mathrm{C}_{11}\right), 59.1$ ( $\left.\mathrm{C}_{6}\right), 129.2$ (C-ar.), 130.1 (C-ar.), 133.2 (C-Cl), 136.9 ( $\mathrm{C}_{\text {ipso }}$ ar. $)$, $149.2\left(C_{3}\right), 171.0\left(C_{1}\right.$ or $\left.C_{5}\right), 172.1\left(C_{1}\right.$ or $\left.C_{5}\right), 207.0\left(C_{9}\right)$.

7,11-Bis(4-chlorophenyl)-2,4-dimethyl-2,4-diazaspiro[5.5] undecane-1,3,5,9-tetraone (7ca'b'). White crystalline solid; m.p. 220-222 ${ }^{\circ} \mathrm{C}$; IR ( $\mathrm{KBr}, \mathrm{cm}^{-1}$ ) 3010 (ar.), 2924 (aliph.), 2854 (aliph.), $1746(\mathrm{C}=\mathrm{O}), 1679(\mathrm{C}=\mathrm{O}), 1492,1421,1379,1091,1015 ;{ }^{1} \mathrm{H}$ NMR $\left(300 \mathrm{MHz}, \mathrm{CDCl}_{3}\right) \delta 2.59$ $\left(\mathrm{dd}, J=14.7 \mathrm{~Hz}, J=3.6 \mathrm{~Hz}, 2 \mathrm{H}\right.$, diastereotopic $\left.-\mathrm{CH}_{2}-\right), 2.92(\mathrm{~s}, 3 \mathrm{H}, \mathrm{MeN}), 3.06(\mathrm{~s}, 3 \mathrm{H}, \mathrm{MeN})$, $3.66\left(\mathrm{t}, 2 \mathrm{H}\right.$, diastereotopic $\left.-\mathrm{CH}_{2}-\right), 3.98(\mathrm{dd}, J=14.1 \mathrm{~Hz}, J=3.6 \mathrm{~Hz}, 2 \mathrm{H},-\mathrm{CH}-\mathrm{ph}$ benzylic proton), $7.00\left(\mathrm{~d}, J=8.1 \mathrm{~Hz}, 4 \mathrm{H}\right.$, ar.), $7.23(\mathrm{~d}, J=8.1 \mathrm{~Hz}, 4 \mathrm{H}$, ar. $) ;{ }^{13} \mathrm{C} \mathrm{NMR}\left(75 \mathrm{MHz}, \mathrm{CDCl}_{3}\right) \delta$ $28.0\left(\mathrm{NCH}_{3}\right), 28.5\left(\mathrm{NCH}_{3}\right), 42.8\left(\mathrm{C}_{8}\right.$ or $\left.\mathrm{C}_{10}\right), 49.8\left(\mathrm{C}_{7}\right.$ or $\left.\mathrm{C}_{11}\right), 60.5\left(\mathrm{C}_{6}\right), 128.8(\mathrm{C}-\mathrm{ar}),. 129.1(\mathrm{C}-$ ar.), $134.6(\mathrm{C}-\mathrm{Cl}), 135.4\left(\mathrm{C}_{\text {ipso }}\right.$ ar. $), 149.3\left(\mathrm{C}_{3}\right), 168.7\left(\mathrm{C}_{1}\right.$ or $\left.\mathrm{C}_{5}\right), 170.4\left(\mathrm{C}_{1}\right.$ or $\left.\mathrm{C}_{5}\right), 207.1\left(\mathrm{C}_{9}\right)$.

7,11-bis(4-chlorophenyl)-3-thioxo-2,4-diazaspiro[5.5] undecane-1,5,9-trione (7ca'c") [46]. Pale yellow crystalline solid; m.p. $200{ }^{\circ} \mathrm{C}$ (decamps.); IR (KBr, cm $\left.{ }^{-1}\right) 3176(\mathrm{NH}), 2918$ (aliph.), 2834 (aliph.), $1726(\mathrm{C}=\mathrm{O}), 1700(\mathrm{C}=\mathrm{O}), 1521,1492,1320,1149,1014,831,519 ;{ }^{1} \mathrm{H}$ NMR (300 MHz, DMSO- $\left.d_{6}\right) \delta 2.44\left(\mathrm{dd}, J=14.7 \mathrm{~Hz}, J=3.6 \mathrm{~Hz}, 2 \mathrm{H}\right.$, diastereotopic $\left.-\mathrm{CH}_{2}-\right), 3.40(\mathrm{~d}, J=$ $13.8 \mathrm{~Hz}, 2 \mathrm{H}$, diastereotopic $\left.-\mathrm{CH}_{2}-\right), 4.00(\mathrm{dd}, J=13.8 \mathrm{~Hz}, J=3.9 \mathrm{~Hz}, 2 \mathrm{H}$, $-\mathrm{CH}-\mathrm{ph}$ benzylic proton), 7.10 (d, $J=8.1 \mathrm{~Hz}, 4 \mathrm{H}$, ar.), 7.37 (d, $J=8.1 \mathrm{~Hz}, 4 \mathrm{H}$, ar.), 12.30 (s, 1H, NH), 12.55 (s, $1 \mathrm{H}, \mathrm{NH}) ;{ }^{13} \mathrm{C}$ NMR $\left(75 \mathrm{MHz}, \mathrm{CDCl}_{3}\right) \delta 43.0\left(\mathrm{C}_{8}\right.$ or $\left.\mathrm{C}_{10}\right), 48.7\left(\mathrm{C}_{7}\right.$ or $\left.\mathrm{C}_{11}\right), 59.7\left(\mathrm{C}_{6}\right), 59.7\left(\mathrm{C}_{6}\right)$, 129.3 (C-ar.), 130.1 (C-ar.), $133.3(\mathrm{C}-\mathrm{Cl}), 136.7$ ( $\mathrm{C}_{\text {ipso }}$ ar. $), 168.7\left(\mathrm{C}_{1}\right.$ or $\left.\mathrm{C}_{5}\right), 170.0\left(\mathrm{C}_{1}\right.$ or $\left.\mathrm{C}_{5}\right)$, $177.8\left(\mathrm{C}_{3}\right), 206.8\left(\mathrm{C}_{9}\right)$.

7,11-Bis(4-chlorophenyl)-2,4-diethyl-3-thioxo-2,4-diazaspiro[5.5] undecane-1,5,9-trione (7ca'd") [46]. Yellow crystalline solid; m.p. 86-90 ${ }^{\circ} \mathrm{C}$; IR (KBr, $\mathrm{cm}^{-1}$ ) 3030 (ar.), 2980 (aliph.), 2933 (aliph.), 1706 (C=O), 1673 (C=O), 1492, 1399, 1114, 1092, 856, 518; ${ }^{1} \mathrm{H}$ NMR (300 MHz, $\left.\mathrm{CDCl}_{3}\right) \delta 1.12\left(\mathrm{t}, J=6.6 \mathrm{~Hz}, 3 \mathrm{H}, \mathrm{NCH}_{2} \mathrm{Me}\right), 1.25\left(\mathrm{t}, J=6.6 \mathrm{~Hz}, 3 \mathrm{H}, \mathrm{NCH}_{2} \mathrm{Me}\right), 2.60(\mathrm{dd}, J=$ $14.9 \mathrm{~Hz}, J=3.9 \mathrm{~Hz}, 2 \mathrm{H}$, diastereotopic $\left.-\mathrm{CH}_{2}-\right), 3.66\left(\mathrm{t}, J=14.4 \mathrm{~Hz}, 2 \mathrm{H}\right.$, diastereotopic $\left.-\mathrm{CH}_{2}-\right)$, 3.98-4.07 (m, $\left.4 \mathrm{H}, 2 \mathrm{NCH}_{2} \mathrm{Me}\right), 4.20-4.30$ (m, $2 \mathrm{H},-\mathrm{CH}-$ ph benzylic proton), $7.01(\mathrm{~d}, J=8.1 \mathrm{~Hz}$, $4 \mathrm{H}$, ar. $), 7.20(\mathrm{~d}, J=8.1 \mathrm{~Hz}, 4 \mathrm{H}$, ar. $) ;{ }^{13} \mathrm{C} \mathrm{NMR}\left(75 \mathrm{MHz}, \mathrm{CDCl}_{3}\right) \delta 11.8\left(\mathrm{CH}_{3}\right), 11.9\left(\mathrm{CH}_{3}\right)$, $43.0\left(\mathrm{NCH}_{2}-\right), 43.3\left(\mathrm{NCH}_{2}-\right), 43.7\left(\mathrm{C}_{8}\right.$ or $\left.\mathrm{C}_{10}\right), 50.4\left(\mathrm{C}_{7}\right.$ or $\left.\mathrm{C}_{11}\right), 60.2\left(\mathrm{C}_{6}\right), 129.0(\mathrm{C}-\mathrm{ar}),$. (C-ar.), $134.6(\mathrm{C}-\mathrm{Cl}), 135.2\left(\mathrm{C}_{\text {ipso }}\right.$ ar. $), 166.7\left(\mathrm{C}_{1}\right.$ or $\left.\mathrm{C}_{5}\right), 168.6\left(\mathrm{C}_{1}\right.$ or $\left.\mathrm{C}_{5}\right), 176.8\left(\mathrm{C}_{3}\right), 207.2\left(\mathrm{C}_{9}\right)$.

7,11-Bis(4-bromophenyl)-2,4-diazaspiro[5.5]undecane-1,3,5,9-tetraone (7ea'a"). Pale yellow crystalline solid; m.p. $220{ }^{\circ} \mathrm{C}$ (decomps.); IR ( $\left.\mathrm{KBr}, \mathrm{cm}^{-1}\right) 3418(\mathrm{OH}$ and/or NH), $3204(\mathrm{NH})$, 3097 (ar.), 2844 (aliph.), 1693 (C=O), 1652 (C=O), 1590, 1328, 1074, 1010, 519; ${ }^{1} \mathrm{H}$ NMR (300 $\left.\mathrm{MHz}, \mathrm{DMSO}-d_{6}\right) \delta 2.5\left(\mathrm{~m}, 2 \mathrm{H}\right.$, diastereotopic $\left.-\mathrm{CH}_{2}-\right), 3.43\left(\mathrm{~m}, 2 \mathrm{H}\right.$, diastereotopic $-\mathrm{CH}_{2^{-}}$, overlapped with DMSO $\left(\mathrm{H}_{2} \mathrm{O}\right)$ peak), 3.97 (d, $J=13.8 \mathrm{~Hz}, 2 \mathrm{H},-\mathrm{CH}-$ ph benzylic proton), 7.04 (d, $J=7.8 \mathrm{~Hz}, 4 \mathrm{H}$, ar.), 7.52 (d, $J=7.8 \mathrm{~Hz}, 4 \mathrm{H}$, ar.), $11.32(\mathrm{bs}, 2 \mathrm{H}, 2 \mathrm{NH}) ;{ }^{13} \mathrm{C} \mathrm{NMR}(75 \mathrm{MHz}$, DMSO- $\left.d_{6}\right) \delta 42.8\left(\mathrm{C}_{8}\right.$ or $\left.\mathrm{C}_{10}\right) 48.5\left(\mathrm{C}_{7}\right.$ or $\left.\mathrm{C}_{11}\right), 59.0\left(\mathrm{C}_{6}\right), 121.8(\mathrm{C}-\mathrm{Br}), 130.5(\mathrm{C}-\mathrm{ar}),. 132.2(\mathrm{C}-$ ar.), $137.3\left(\mathrm{C}_{\text {ipso }}\right.$ ar. $), 149.2\left(\mathrm{C}_{3}\right), 171.0\left(\mathrm{C}_{1}\right.$ or $\left.\mathrm{C}_{5}\right), 172.0\left(\mathrm{C}_{1}\right.$ or $\left.\mathrm{C}_{5}\right), 207.0\left(\mathrm{C}_{9}\right)$. 
7,11-Bis(4-bromophenyl)-2,4-dimethyl-2,4-diazaspiro[5.5] undecane-1,3,5,9-tetraone (7ea'b'). White crystalline solid; m.p. 237-239 ${ }^{\circ} \mathrm{C}$; IR (KBr, $\mathrm{cm}^{-1}$ ) 3088 (ar.), 2973 (aliph.), 2925 (aliph.), $1715(\mathrm{C}=\mathrm{O}), 1679(\mathrm{C}=\mathrm{O}), 1530,1378,1350,1073,509 ;{ }^{1} \mathrm{H}$ NMR $\left(300 \mathrm{MHz}, \mathrm{CDCl}_{3}\right) \delta 2.57$ $\left(\mathrm{dd}, J=14.7 \mathrm{~Hz}, J=3.9 \mathrm{~Hz}, 2 \mathrm{H}\right.$, diastereotopic $\left.-\mathrm{CH}_{2}-\right), 2.90(\mathrm{~s}, 3 \mathrm{H}, \mathrm{MeN}), 3.05(\mathrm{~s}, 3 \mathrm{H}, \mathrm{MeN})$, $3.64\left(\mathrm{t}, 2 \mathrm{H}\right.$, diastereotopic $\left.-\mathrm{CH}_{2}-\right), 3.97(\mathrm{dd}, J=14.4 \mathrm{~Hz}, J=4.2 \mathrm{~Hz}, 2 \mathrm{H},-\mathrm{CH}-\mathrm{ph}$ benzylic proton), 6.93 (d, $J=8.1 \mathrm{~Hz}, 4 \mathrm{H}$, ar.), 7.37 (d, $J=8.1 \mathrm{~Hz}, 4 \mathrm{H}$, ar.); ${ }^{13} \mathrm{C} \mathrm{NMR}\left(75 \mathrm{MHz}, \mathrm{CDCl}_{3}\right) \delta$ $28.1\left(\mathrm{NCH}_{3}\right), 28.5\left(\mathrm{NCH}_{3}\right), 42.7\left(\mathrm{C}_{8}\right.$ or $\left.\mathrm{C}_{10}\right), 49.8\left(\mathrm{C}_{7}\right.$ or $\left.\mathrm{C}_{11}\right), 60.3\left(\mathrm{C}_{6}\right), 122.8(\mathrm{C}-\mathrm{Br}), 129.2(\mathrm{C}-$ ar.), 132.1 (C-ar.), 136.0 ( $\mathrm{C}_{\text {ipso }}$ ar. $), 149.3\left(\mathrm{C}_{3}\right), 168.6\left(\mathrm{C}_{1}\right.$ or $\left.\mathrm{C}_{5}\right), 170.3\left(\mathrm{C}_{1}\right.$ or $\left.\mathrm{C}_{5}\right), 207.0\left(\mathrm{C}_{9}\right)$.

7,11-Bis(4-Bromophenyl)-3-thioxo-2,4-diazaspiro[5.5] undecane-1,5,9-trione (7ea'c') [46]. Yellow crystalline solid; m.p. $200{ }^{\circ} \mathrm{C}$ (decomps.); IR (KBr, cm${ }^{-1}$ ) 3189 (NH), 2919 (aliph.), $1701(\mathrm{C}=\mathrm{O}), 1521,1321,1148,1009,829,515 ;{ }^{1} \mathrm{H}$ NMR $\left(300 \mathrm{MHz}\right.$, DMSO- $\left.d_{6}\right) \delta 2.43(\mathrm{~m}, 2 \mathrm{H}$, diastereotopic $\left.-\mathrm{CH}_{2}{ }^{-}\right), 3.46\left(\mathrm{~m}, 2 \mathrm{H}\right.$, diastereotopic $\left.-\mathrm{CH}_{2}-\right), 4.00(\mathrm{dd}, J=13.2 \mathrm{~Hz}, J=4.5 \mathrm{~Hz}, 2 \mathrm{H}$, -CH-ph benzylic proton), 7.03 (d, $J=8.1 \mathrm{~Hz}, 4 \mathrm{H}$, ar.), 7.50 (d, $J=8.1 \mathrm{~Hz}, 4 \mathrm{H}$, ar.), 12.30 (s, $1 \mathrm{H}, \mathrm{NH}), 12.55(\mathrm{~s}, 1 \mathrm{H}, \mathrm{NH}) ;{ }^{13} \mathrm{C}$ NMR $\left(75 \mathrm{MHz}, \mathrm{DMSO}-d_{6}\right) \delta 42.9\left(\mathrm{C}_{8}\right.$ or $\left.\mathrm{C}_{10}\right), 48.8\left(\mathrm{C}_{7}\right.$ or $\left.\mathrm{C}_{11}\right)$, $59.5\left(\mathrm{C}_{6}\right), 121.9$ (C-Br), 130.4 (C-ar.), 132.2 (C-ar.), 137.1 ( $\mathrm{C}_{\text {ipso }}$ ar. $), 168.7\left(\mathrm{C}_{1}\right.$ or $\left.\mathrm{C}_{5}\right), 170.0\left(\mathrm{C}_{1}\right.$ or $\left.\mathrm{C}_{5}\right), 177.8\left(\mathrm{C}_{3}\right), 206.7\left(\mathrm{C}_{9}\right)$.

7,11-Bis(4-bromophenyl)-2,4-diethyl-3-thioxo-2,4-diazaspiro[5.5] undecane-1,5,9-trione

(7ea'd") [46]. Yellow crystalline solid; m.p. 173-175 ${ }^{\circ} \mathrm{C}$; IR $\left(\mathrm{KBr}, \mathrm{cm}^{-1}\right) 3050$ (ar.), 2980 (aliph.), 2932 (aliph.), 1704 (C=O), 1673 (C=O), 1489, 1398, 1291, 1114, 855, 515; ${ }^{1} \mathrm{H}$ NMR $\left(300 \mathrm{MHz}, \mathrm{CDCl}_{3}\right) \delta 0.90\left(\mathrm{t}, J=6.9 \mathrm{~Hz}, 3 \mathrm{H}, \mathrm{NCH}_{2} \mathrm{Me}\right), 1.11\left(\mathrm{t}, J=6.6 \mathrm{~Hz}, 3 \mathrm{H}, \mathrm{NCH}_{2} \mathrm{Me}\right), 2.60$ $\left(\mathrm{dd}, J=15.0 \mathrm{~Hz}, J=3.9 \mathrm{~Hz}, 2 \mathrm{H}\right.$, diastereotopic $\left.-\mathrm{CH}_{2}-\right), 3.67(\mathrm{t}, J=14.4 \mathrm{~Hz}, 2 \mathrm{H}$, diastereotopic $-\mathrm{CH}_{2}$ ) $, 3.97-4.18\left(\mathrm{~m}, 4 \mathrm{H}, 2 \mathrm{NCH}_{2} \mathrm{Me}\right), 4.20$ (m, 2H, $-\mathrm{CH}-\mathrm{ph}$ benzylic proton), 6.96 (d, $J=8.1$ $\mathrm{Hz}, 4 \mathrm{H}$, ar.), 7.37 (d, $J=8.1 \mathrm{~Hz}, 4 \mathrm{H}$, ar.); ${ }^{13} \mathrm{C} \mathrm{NMR}\left(75 \mathrm{MHz}, \mathrm{CDCl}_{3}\right) \delta 11.8\left(\mathrm{NCH}_{3}\right), 12.0$ $\left(\mathrm{NCH}_{3}\right), 42.9\left(\mathrm{NCH}_{2}-\right), 43.3\left(\mathrm{NCH}_{2}-\right), 43.7\left(\mathrm{C}_{8}\right.$ or $\left.\mathrm{C}_{10}\right), 50.5\left(\mathrm{C}_{7}\right.$ or $\left.\mathrm{C}_{11}\right), 60.0\left(\mathrm{C}_{6}\right), 122.7(\mathrm{C}-\mathrm{Br})$, 129.5 (C-ar.), 132.0 (C-ar.), 135.7 ( $\mathrm{C}_{\text {ipso }}$ ar.), $166.7\left(\mathrm{C}_{1}\right.$ or $\left.\mathrm{C}_{5}\right), 168.6\left(\mathrm{C}_{1}\right.$ or $\left.\mathrm{C}_{5}\right), 176.8\left(\mathrm{C}_{3}\right)$, $207.1\left(\mathrm{C}_{9}\right)$.

7,11-Diphenyl-2,4-diazaspiro[5.5] undecane-1,3,5,9-tetraone (7fa'a') [36]. White crystalline solid; m.p. 297-299 ${ }^{\circ} \mathrm{C}$ (decomps.); IR (KBr, $\left.\mathrm{cm}^{-1}\right) 3201$ (NH), 3087 (ar.), 2928 (aliph.), 2855 (aliph.), 1755 (C=O), $1708(\mathrm{C}=\mathrm{O}), 1683(\mathrm{C}=\mathrm{O}), 1411,1377,1242,704 ;{ }^{1} \mathrm{H}$ NMR $(300 \mathrm{MHz}$, DMSO- $\left.d_{6}\right) \delta 2.42\left(\mathrm{~d}, J=4.2 \mathrm{~Hz}, 2 \mathrm{H}\right.$, diastereotopic $\left.-\mathrm{CH}_{2^{-}}\right), 3.52(\mathrm{t}, J=14.1 \mathrm{~Hz}, 2 \mathrm{H}$, diastereotopic $\left.-\mathrm{CH}_{2}-\right), 3.96(\mathrm{dd}, J=13.8 \mathrm{~Hz}, J=4.5 \mathrm{~Hz}, 2 \mathrm{H},-\mathrm{CH}-$ ph benzylic proton), 7.12 (d, $J$ $=6.3 \mathrm{~Hz}, 4 \mathrm{H}$, ar. $), 7.26-7.31(\mathrm{~m}, 6 \mathrm{H}, \mathrm{ar}),. 11.14(\mathrm{~s}, 1 \mathrm{H}, \mathrm{NH}), 11.40(\mathrm{~s}, 1 \mathrm{H}, \mathrm{NH}) ;{ }^{13} \mathrm{C} \mathrm{NMR}(75$ MHz, DMSO- $\left.d_{6}\right) \delta 43.2\left(\mathrm{C}_{8}\right.$ or $\left.\mathrm{C}_{10}\right), 49.3\left(\mathrm{C}_{7}\right.$ or $\left.\mathrm{C}_{11}\right), 59.3\left(\mathrm{C}_{6}\right), 128.2$ (C-ar.), 128.5 (C-ar.), 129.2 (C-ar.), $138.0\left(\mathrm{C}_{\text {ipso }}\right.$ ar. $), 149.2\left(\mathrm{C}_{3}\right), 171.3\left(\mathrm{C}_{1}\right.$ or $\left.\mathrm{C}_{5}\right), 172.2\left(\mathrm{C}_{1}\right.$ or $\left.\mathrm{C}_{5}\right), 207.6\left(\mathrm{C}_{9}\right)$; $\mathrm{MS}$ $(\mathrm{m} / \mathrm{z}, \%), 362\left(\mathrm{M}^{+}, 65\right), 258$ (25), 230 (90), 172 (28), 146 (50), 129 (18), 104 (100, base peak), 77 (40), $51(15)$.

7,11-Diphenyl-3-thioxo-2,4-diazaspiro[5.5]undecane-1,5,9-trione (7fa'c') [46]. Yellow crystalline solid; m.p. $253-255{ }^{\circ} \mathrm{C}$; IR $\left(\mathrm{KBr}, \mathrm{cm}^{-1}\right) 3438(\mathrm{OH}$ and/or NH), $3140(\mathrm{NH}), 2927$ (aliph.), $1709(\mathrm{C}=\mathrm{O}), 1680(\mathrm{C}=\mathrm{O}), 1536,1333,1148,702.9 ;{ }^{1} \mathrm{H}$ NMR (300 MHz, DMSO- $\left.d_{6}\right) \delta$ $2.49\left(\mathrm{~d}, J=4.5 \mathrm{~Hz}, 2 \mathrm{H}\right.$, diastereotopic $\left.-\mathrm{CH}_{2}\right), 3.49\left(\mathrm{t}, J=14.1 \mathrm{~Hz}, 2 \mathrm{H}\right.$, diastereotopic $\left.-\mathrm{CH}_{2}-\right)$, 3.96 (dd, $J=14.1 \mathrm{~Hz}, J=4.5 \mathrm{~Hz}, 2 \mathrm{H},-\mathrm{CH}-$ ph benzylic proton), 7.11 (d, $J=6.6 \mathrm{~Hz}, 4 \mathrm{H}$, ar.), 7.29 (d, $J=6.6 \mathrm{~Hz}, 4 \mathrm{H}$, ar. $), 12.15$ (s, $1 \mathrm{H}, \mathrm{NH}), 12.43$ (s, 1H, NH); ${ }^{13} \mathrm{C}$ NMR (75 MHz, DMSO$\left.d_{6}\right) \delta 43.3\left(\mathrm{C}_{8}\right.$ or $\left.\mathrm{C}_{10}\right), 49.6\left(\mathrm{C}_{7}\right.$ or $\left.\mathrm{C}_{11}\right), 59.8\left(\mathrm{C}_{6}\right), 128.2$ (C-ar.), 128.6 (C-ar.), 129.3 (C-ar.), 137.8 ( $\mathrm{C}_{\text {ipso }}$ ar. $), 169.1\left(\mathrm{C}_{1}\right.$ or $\left.\mathrm{C}_{5}\right), 170.3\left(\mathrm{C}_{1}\right.$ or $\left.\mathrm{C}_{5}\right), 177.9\left(\mathrm{C}_{3}\right), 207.4\left(\mathrm{C}_{9}\right)$.

2,4-Diethyl-7,11-diphenyl-3-thioxo-2,4-diazaspiro[5.5]undecane-1,5,9-trione (7fa'd") [46]. Yellow crystalline solid; m.p. 130-132 ${ }^{\circ} \mathrm{C}$; IR (KBr, cm $\left.{ }^{-1}\right) 3033$ (ar.), 2979 (aliph.), 2931 (aliph.), 1707 (C=O), 1672 (C=O), 1398, 1290, 1113, 700; ${ }^{1} \mathrm{H}$ NMR (300 MHz, $\left.\mathrm{CDCl}_{3}\right) \delta 0.87$ 
$\left(\mathrm{t}, J=7.2 \mathrm{~Hz}, 3 \mathrm{H}, \mathrm{NCH}_{2} \mathrm{Me}\right), 1.09\left(\mathrm{t}, J=6.9 \mathrm{~Hz}, 3 \mathrm{H}, \mathrm{NCH}_{2} \mathrm{Me}\right), 2.65(\mathrm{dd}, J=15.0 \mathrm{~Hz}, J=3.6$ $\mathrm{Hz}, 2 \mathrm{H}$, diastereotopic $\left.-\mathrm{CH}_{2}-\right), 3.75\left(\mathrm{t}, J=14.7 \mathrm{~Hz}, 2 \mathrm{H}\right.$, diastereotopic $\left.-\mathrm{CH}_{2^{-}}\right), 3.98-4.09(\mathrm{~m}, 4 \mathrm{H}$, $2 \mathrm{NCH}_{2} \mathrm{Me}$ ), 4.18 (q, $J=6.9 \mathrm{~Hz}, 2 \mathrm{H},-\mathrm{CH}$-ph benzylic proton), 7.02-7.23 (m, $\left.10 \mathrm{H}, \mathrm{ar}.\right) ;{ }^{13} \mathrm{C}$ $\operatorname{NMR}\left(75 \mathrm{MHz}, \mathrm{CDCl}_{3}\right) \delta 11.7\left(\mathrm{CH}_{3}\right), 11.8\left(\mathrm{CH}_{3}\right), 43.1\left(\mathrm{C}_{8}\right.$ or $\left.\mathrm{C}_{10}\right), 43.2\left(\mathrm{C}_{7}\right.$ or $\left.\mathrm{C}_{11}\right), 43.6$ $\left.\left(\mathrm{NCH}_{2}\right)^{-}\right), 51.1\left(\mathrm{NCH}_{2^{-}}\right), 60.6\left(\mathrm{C}_{6}\right), 127.6$ (C-ar.), 128.6 (C-ar.), 128.8 (C-ar.), 136.8 ( $\mathrm{C}_{\text {ipso }}$ ar. $)$, $167.1\left(\mathrm{C}_{1}\right.$ or $\left.\mathrm{C}_{5}\right), 169.0\left(\mathrm{C}_{1}\right.$ or $\left.\mathrm{C}_{5}\right), 177.3\left(\mathrm{C}_{3}\right), 208.2\left(\mathrm{C}_{9}\right)$; $\mathrm{MS}(\mathrm{m} / z, \%), 434\left(\mathrm{M}^{+}, 6\right), 390(12)$, 330 (65), 305 (8), 287 (25), 269 (70), 235 (40), 216 (10), 187 (20), 167 (18), 146 (85), 123 (20), 97 (50), 69 (100, base peak), 41 (27).

7,11-Bis(4-methoxyphenyl)-2,4-diazaspiro[5.5] undecane-1,3,5,9-tetraone (7ga'a"). White crystalline solid; m.p. $150{ }^{\circ} \mathrm{C}$ (decomps.); IR (KBr, cm ${ }^{-1}$ ) 3228 (NH), 3103 (NH), 2963 (aliph.), 2838 (aliph.), $1710(\mathrm{C}=\mathrm{O}), 1611,1513,1252,1031,836 ;{ }^{1} \mathrm{H}$ NMR $\left(300 \mathrm{MHz}, \mathrm{DMSO}-d_{6}\right) \delta 2.36$ (d, $J=13.8 \mathrm{~Hz}, 2 \mathrm{H}$, diastereotopic $\left.-\mathrm{CH}_{2}-\right), 3.46\left(\mathrm{t}, J=15.0 \mathrm{~Hz}, 2 \mathrm{H}\right.$, diastereotopic $-\mathrm{CH}_{2}$ ), 3.66 (s, 6H, 2MeO), 3.84 (d, $J=12.9 \mathrm{~Hz}, 2 \mathrm{H},-\mathrm{CH}$-ph benzylic proton), 6.83 (d, $J=7.2 \mathrm{~Hz}, 4 \mathrm{H}$, ar.), $7.00(\mathrm{~d}, J=7.2 \mathrm{~Hz}, 4 \mathrm{H}$, ar.), 7.83 (bs, $2 \mathrm{H}, 2 \mathrm{NH}) ;{ }^{13} \mathrm{C}$ NMR $\left(75 \mathrm{MHz}\right.$, DMSO- $\left.d_{6}\right) \delta 43.5\left(\mathrm{C}_{8}\right.$ or $\left.\mathrm{C}_{10}\right), 48.5\left(\mathrm{C}_{7}\right.$ or $\left.\mathrm{C}_{11}\right), 55.4\left(\mathrm{C}_{6}\right), 59.6\left(\mathrm{OCH}_{3}\right), 114.4$ (C-ar.), 130.0 (C-ar.), 130.7 ( $\mathrm{C}_{\text {ipso }}$ ar. $)$, $149.7\left(\mathrm{C}_{3}\right), 159.2\left(\mathrm{C}-\mathrm{OCH}_{3}\right), 171.6\left(\mathrm{C}_{1}\right.$ or $\left.\mathrm{C}_{5}\right), 172.7\left(\mathrm{C}_{1}\right.$ or $\left.\mathrm{C}_{5}\right), 208.0\left(\mathrm{C}_{9}\right)$.

7,11-Bis(4-methoxyphenyl)-2,4-dimethyl-2,4-diazaspiro[5.5] undecane-1,3,5,9-tetraone

$\left(7 \boldsymbol{g} \boldsymbol{a}^{\prime} \boldsymbol{b}^{\prime \prime}\right)$. White crystalline solid; m.p. $65-67{ }^{\circ} \mathrm{C}$; IR (KBr, cm $\left.{ }^{-1}\right) 3020$ (ar.), 2956 (aliph.), 2837 (aliph.), $1716(\mathrm{C}=\mathrm{O}), 1676(\mathrm{C}=\mathrm{O}), 1601,1512,1423,1307,1253,1032,833,753 ;{ }^{1} \mathrm{H}$ NMR $\left.\left(300 \mathrm{MHz}, \mathrm{CDCl}_{3}\right) \delta 2.58\left(\mathrm{dd}, J=14.4 \mathrm{~Hz}, J=3.6 \mathrm{~Hz}, 2 \mathrm{H} \text {, diastereotopic }-\mathrm{CH}_{2}\right)^{-}\right), 2.91(\mathrm{~s}, 3 \mathrm{H}$, $\mathrm{MeN}), 3.04(\mathrm{~s}, 3 \mathrm{H}, \mathrm{MeN}), 3.76(\mathrm{~s}, 6 \mathrm{H}, 2 \mathrm{MeO}), 3.63-3.99\left(\mathrm{~m}, 4 \mathrm{H}\right.$, diastereotopic $-\mathrm{CH}_{2}$ - and $-\mathrm{CH}-$ ph benzylic proton), 6.75 (d, $J=8.7 \mathrm{~Hz}, 4 \mathrm{H}$, ar.), 6.99 (d, $J=8.7 \mathrm{~Hz}, 4 \mathrm{H}$, ar.); ${ }^{13} \mathrm{C}$ NMR $(75$ $\left.\mathrm{MHz}, \mathrm{CDCl}_{3}\right) \delta 27.9\left(\mathrm{NCH}_{3}\right), 28.4\left(\mathrm{NCH}_{3}\right), 43.2\left(\mathrm{C}_{8}\right.$ or $\left.\mathrm{C}_{10}\right), 49.7\left(\mathrm{C}_{7}\right.$ or $\left.\mathrm{C}_{11}\right), 55.2\left(\mathrm{C}_{6}\right), 61.3$ $\left(\mathrm{OCH}_{3}\right), 114.1$ (C-ar.), 128.6 (C-ar.), 130.1 ( $\left.\mathrm{C}_{\text {ipso-ar. }}\right), 159.4\left(\mathrm{C}_{3}\right), 165.0\left(\mathrm{C}-\mathrm{OCH}_{3}\right), 169.2\left(\mathrm{C}_{1}\right.$ or $\left.\mathrm{C}_{5}\right), 170.9\left(\mathrm{C}_{1}\right.$ or $\left.\mathrm{C}_{5}\right), 208.5\left(\mathrm{C}_{9}\right)$.

2,4-Diethyl-7,11-bis(4-methoxyphenyl)-3-thioxo-2,4-diazaspiro[5.5] undecane-1,5,9-trione (7ga'd") [46]. Yellow crystalline solid; m.p. $120^{\circ} \mathrm{C}$; IR (KBr, $\left.\mathrm{cm}^{-1}\right) 3010$ (ar.), 2976 (aliph.), 2931 (aliph.), 2837 (aliph.), 1706 (C=O), 1675 (C=O), 1594, 1512, 1399, 1255, 1174, 1105, $1031,833,532 ;{ }^{1} \mathrm{H}$ NMR $\left(300 \mathrm{MHz}, \mathrm{CDCl}_{3}\right) \delta 0.91\left(\mathrm{t}, J=6.9 \mathrm{~Hz}, 3 \mathrm{H}, \mathrm{NCH}_{2} \mathrm{Me}\right), 1.11(\mathrm{t}, J=$ $\left.6.9 \mathrm{~Hz}, 3 \mathrm{H}, \mathrm{NCH}_{2} \mathrm{Me}\right), 1.24-1.43\left(\mathrm{~m}, 2 \mathrm{H}\right.$, diastereotopic $\left.-\mathrm{CH}_{2}-\right), 2.58(\mathrm{dd}, J=14.7 \mathrm{~Hz}, J=3.9$ $\mathrm{Hz}, 2 \mathrm{H}$, diastereotopic $\left.-\mathrm{CH}_{2}-\right), 3.71\left(\mathrm{t}, J=14.7 \mathrm{~Hz}, 2 \mathrm{H}\right.$, diastereotopic $\left.-\mathrm{CH}_{2}-\right), 3.74(\mathrm{~s}, 6 \mathrm{H}$, 2MeO), 3.87-4.08 (m, $\left.4 \mathrm{H}, 2 \mathrm{NCH}_{2} \mathrm{Me}\right), 4.20(\mathrm{dd}, J=13.5 \mathrm{~Hz}, J=6.6 \mathrm{~Hz}, 2 \mathrm{H},-\mathrm{CH}-$ ph benzylic proton), $6.74(\mathrm{~d}, J=8.7 \mathrm{~Hz}, 4 \mathrm{H}$, ar. $), 6.99(\mathrm{~d}, J=8.7 \mathrm{~Hz}, 4 \mathrm{H}, \mathrm{ar}.) ;{ }^{13} \mathrm{C}$ NMR $\left(75 \mathrm{MHz}, \mathrm{CDCl}_{3}\right) \delta$ $11.8\left(\mathrm{CH}_{3}\right), 11.9\left(\mathrm{CH}_{3}\right), 43.2\left(\mathrm{C}_{8}\right.$ or $\left.\mathrm{C}_{10}\right), 43.4\left(\mathrm{C}_{7}\right.$ or $\left.\mathrm{C}_{11}\right), 43.6\left(\mathrm{NCH}_{2}-\right), 50.3\left(\mathrm{NCH}_{2}\right), 55.3$ $\left(\mathrm{C}_{6}\right), 61.1\left(\mathrm{OCH}_{3}\right), 114.1$ (C-ar.), 123.4 (C-ar.), 128.9 ( $\mathrm{C}_{\text {ipso }}$ ar. $), 159.5\left(\mathrm{C}-\mathrm{OCH}_{3}\right), 167.2\left(\mathrm{C}_{1}\right.$ or $\left.\mathrm{C}_{5}\right), 169.2\left(\mathrm{C}_{1}\right.$ or $\left.\mathrm{C}_{5}\right), 177.4\left(\mathrm{C}_{3}\right), 208.6\left(\mathrm{C}_{9}\right)$.

4-Bis(4-bromophenyl)-1'H-spiro[bicyclo[3.3.1]nonane-3,5'-pyrimidine]-4',6',9-trione (mixture of two diastereomers, $\left.7 \boldsymbol{e} \boldsymbol{b}^{\prime} \boldsymbol{b}^{\prime \prime}\right)$. In a $50 \mathrm{~mL}$ round bottom flask equipped with magnetically stirrer the mixture of 1,3-dimethylbarbituric acid (1.3 mmol, $0.21 \mathrm{~g}),(2 E, 6 E)-2,6-$ dibenzylidenecyclohexanone ( $1.3 \mathrm{mmol}, 0.36 \mathrm{~g})$ and triethylamine $(2.1 \mathrm{mmol}, 0.22 \mathrm{~g}, 0.3 \mathrm{~mL})$ in $10 \mathrm{~mL}$ ethanol and refluxed for $24 \mathrm{~h}$. The yellow color disappeared and consequently white crystalline solid precipitated, filtered off, washed with few $\mathrm{mL}$ of cold ethanol then dried $(0.28$ g, 50\%). White crystalline solid; m.p. $262{ }^{\circ} \mathrm{C}$; IR (KBr, cm $\left.{ }^{-1}\right) 3010$ (ar.), 2925 (aliph.), 2854 (aliph.), $1743(\mathrm{C}=\mathrm{O}), 1672(\mathrm{C}=\mathrm{O}), 1577,1450,1383,751,461 ;{ }^{1} \mathrm{H} \mathrm{NMR}\left(300 \mathrm{MHz}, \mathrm{CDCl}_{3}\right) \delta$ $1.2\left(\mathrm{~m}, 1 \mathrm{H}\right.$, diastereotopic $\left.-\mathrm{CH}_{2}-\right), 1.60\left(\mathrm{~m}, 2 \mathrm{H}\right.$, diastereotopic $\left.-\mathrm{CH}_{2}-\right), 1.79-1.82(\mathrm{~m}, 2 \mathrm{H}$, diastereotopic $\left.-\mathrm{CH}_{2}-\right), 2.95\left(\mathrm{~m}, 2 \mathrm{H}\right.$, diastereotopic $\left.-\mathrm{CH}_{2}-\right), 3.03(\mathrm{~s}, 3 \mathrm{H}, \mathrm{MeN}), 3.21(\mathrm{~s}, 3 \mathrm{H}$, $\mathrm{MeN}), 3.71\left(\mathrm{~m}, 1 \mathrm{H}\right.$, diastereotopic $\left.-\mathrm{CH}_{2}-\right), 3.89(\mathrm{~m}, 1 \mathrm{H},-\mathrm{CH}-\mathrm{ph}$ benzylic proton), $4.43(\mathrm{~m}, 1 \mathrm{H},-$ $\mathrm{CH}-\mathrm{ph}$ benzylic proton), 7.06-7.80 (m, 10H, ar.); ${ }^{13} \mathrm{C} \mathrm{NMR}\left(75 \mathrm{MHz}, \mathrm{CDCl}_{3}\right) \delta 214.0\left(\mathrm{C}_{9}\right)$, 
$194.0\left(\mathrm{C}_{9}\right), 168.4\left(\mathrm{C}_{4^{\prime}}\right.$ or $\left.\mathrm{C}_{6^{\prime}}\right), 168.1\left(\mathrm{C}_{4^{\prime}}\right.$ or $\left.\mathrm{C}_{6^{\prime}}\right), 151.1\left(\mathrm{C}_{2^{\prime}}\right), 137.0\left(\mathrm{C}_{\text {ipso }}\right.$ ar. $), 136.2\left(\mathrm{C}_{\text {ipso-ar. }}\right)$, 136.0 (C-ar.), 130.4 (C-ar.), 130.3 (C-ar.), 128.8 (C-ar.), 128.6 (C-Br), 128.4 (C-Br), $51.3\left(\mathrm{C}_{3}\right.$ or $\left.\mathrm{C}_{5}\right)$, $51.1\left(\mathrm{C}_{3}\right.$ or $\left.\mathrm{C}_{5^{\prime}}\right), 49.2,40.8\left(\mathrm{C}_{1}\right.$ or $\left.\mathrm{C}_{5}\right)$, $35.6\left(\mathrm{C}_{1}\right.$ or $\left.\mathrm{C}_{5}\right), 28.5\left(\mathrm{C}_{2}\right.$ or $\left.\mathrm{C}_{4}\right), 28.11\left(\mathrm{C}_{2}\right.$ or $\left.\mathrm{C}_{4}\right)$, $28.06\left(\mathrm{C}_{2}\right.$ or $\left.\mathrm{C}_{4}\right), 26.5\left(\mathrm{NCH}_{3}\right), 25.1\left(\mathrm{NCH}_{3}\right), 23.0\left(\mathrm{C}_{6}\right.$ or $\left.\mathrm{C}_{8}\right), 20.5\left(\mathrm{C}_{7}\right)$.

1',3'-Dimethyl-2,4-bis(3-nitrophenyl)-1'H-spiro[bicyclo[3.3.1]nonane-3,5'-pyrimidine]-2',4',6',9 $\left(3^{\prime} \mathrm{H}\right)$-tetraone (mixture of two diastereomers, $\left.\mathbf{7} \boldsymbol{a b}^{\prime} \boldsymbol{b}^{\prime \prime}\right)$. White crystalline solid; m.p. $66-68{ }^{\circ} \mathrm{C}$; IR ( $\left.\mathrm{KBr}, \mathrm{cm}^{-1}\right) 3082$ (ar.), 2953 (aliph.), 2863 (aliph.), 1693 (C=O), 1527, 1434, 1351, 1047, 811, 744, 682; ${ }^{1} \mathrm{H}$ NMR $\left(300 \mathrm{MHz}, \mathrm{CDCl}_{3}\right) \delta 1.58\left(\mathrm{~m}, 2 \mathrm{H}\right.$, diastereotopic - $\left.\mathrm{CH}_{2}-\right), 1.90-2.10(\mathrm{~m}$, $4 \mathrm{H}$, diastereotopic $\left.-\mathrm{CH}_{2}-\right), 2.66(\mathrm{~s}, 3 \mathrm{H}, \mathrm{MeN}), 2.82-2.98\left(\mathrm{~m}, 3 \mathrm{H}\right.$, diastereotopic $\left.-\mathrm{CH}_{2}-\right), 3.46$, $(\mathrm{s}$, $3 \mathrm{H}, \mathrm{MeN}), 4.65$ (s, 1H, -CH-ph benzylic proton), 6.97 (s, $1 \mathrm{H}$, ar.), 7.43-8.33 (m, $7 \mathrm{H}$, ar.); ${ }^{13} \mathrm{C}$ $\operatorname{NMR}\left(75 \mathrm{MHz}, \mathrm{CDCl}_{3}\right) \delta 22.4\left(\mathrm{C}_{7}\right), 23.2\left(\mathrm{C}_{7}\right), 26.5\left(\mathrm{C}_{6}\right.$ or $\left.\mathrm{C}_{8}\right), 28.2\left(\mathrm{C}_{6}\right.$ or $\left.\mathrm{C}_{8}\right), 28.4\left(\mathrm{C}_{2}\right.$ or $\left.\mathrm{C}_{4}\right)$, $29.5\left(\mathrm{C}_{1}\right.$ or $\mathrm{C}_{5}$ ), $64.6\left(\mathrm{C}_{3}\right.$ or $\left.\mathrm{C}_{5}\right), 123.7$ (C-ar.), 124.0 (C-ar.), 124.4 (C-ar.), 129.0 (C-ar.), 129.2 (C-ar.), 129.3 (C-ar.), 129.9 (C-ar.), 134.7 (C-ar.), 135.0 ( $\mathrm{C}_{i p s o}$-ar.), 135.3 (C $\mathrm{C}_{\text {ipso }}$-ar.), 136.1 (C$\left.\left.\mathrm{NO}_{2}\right), 137.0\left(\mathrm{C}^{-N}\right)_{2}\right), 146.0\left(\mathrm{C}_{2^{\prime}}\right), 150.0\left(\mathrm{C}_{2^{\prime}}\right), 167.0\left(\mathrm{C}_{4^{\prime}}\right.$ or $\left.\mathrm{C}_{6^{\prime}}\right), 167.2\left(\mathrm{C}_{4^{\prime}}\right.$ or $\left.\mathrm{C}_{6^{\prime}}\right), 190.3\left(\mathrm{C}_{9}\right)$, $213.5\left(\mathrm{C}_{9}\right)$.

2,4-Diphenyl-1'H-spiro[bicyclo[3.3.1]nonane-3,5'-pyrimidine]-2',4',6',9(3'H)-tetraone (7fb' $\left.\boldsymbol{a}^{\prime \prime}\right)$. White crystalline solid; m.p. $150{ }^{\circ} \mathrm{C}$ (decomps.); IR ( $\left.\mathrm{KBr}, \mathrm{cm}^{-1}\right) 3423(\mathrm{NH}$ and/or $\mathrm{OH}), 3160$ (NH), 2988 (aliph.), 2941 (aliph.), 2845, 1684 (C=O), 1583, 1447, 1372, 1158, 776, 697, 528; ${ }^{1} \mathrm{H}$ NMR (300 MHz, DMSO- $\left.d_{6}\right) \delta 1.09\left(\mathrm{~m}, 3 \mathrm{H}\right.$, diastereotopic $\left.-\mathrm{CH}_{2}-\right), 1.69(\mathrm{~m}, 2 \mathrm{H}$, diastereotopic $\left.-\mathrm{CH}_{2-}\right), 2.88\left(\mathrm{~m}, 5 \mathrm{H}\right.$, diastereotopic $-\mathrm{CH}_{2^{-}}$and - $\mathrm{CH}$-ph benzylic proton), 7.037.61 (m, 10H, ar.), 8.50, 8.70 (bs, $1 \mathrm{H}, \mathrm{NH}), 10.00$ (s, 1H, NH); ${ }^{13} \mathrm{C}$ NMR (75 MHz, DMSO- $\left.d_{6}\right) \delta$ $21.0\left(\mathrm{C}_{7}\right), 22.9\left(\mathrm{C}_{6}\right.$ or $\left.\mathrm{C}_{8}\right), 28.3\left(\mathrm{C}_{2}\right.$ or $\left.\mathrm{C}_{4}\right), 46.1\left(\mathrm{C}_{1}\right.$ or $\left.\mathrm{C}_{5}\right), 51\left(\mathrm{C}_{3}\right.$ or $\left.\mathrm{C}_{5}\right), 129.0(\mathrm{C}-\mathrm{ar}$ ), 129.3 (C-ar.), 130.8 (C-ar.), 136.2 ( $\mathrm{C}_{\text {ipso }}$-ar. $), 162.0\left(\mathrm{C}_{2^{\prime}}\right), 175.0\left(\mathrm{C}_{4^{\prime}}\right.$ or $\left.\mathrm{C}_{6^{\prime}}\right), 189.4\left(\mathrm{C}_{4^{\prime}}\right.$ or $\left.\mathrm{C}_{6^{\prime}}\right), 213.0$ $\left(\mathrm{C}_{9}\right)$.

1',3'-Dimethyl-2,4-diphenyl-1'H-spiro[bicyclo[3.3.1]nonane-3,5'-pyrimidine]-2',4',6',9(3'H)tetraone (mixture of two diastereomers, $\left.7 f^{\prime} b^{\prime \prime}\right)$. White crystalline solid; m.p. $66-68{ }^{\circ} \mathrm{C}$; IR $\left(\mathrm{KBr}, \mathrm{cm}^{-1}\right) 3050$ (ar.), 2925 (aliph.), 2854 (aliph.), 1743 (C=O), $1672(\mathrm{C}=\mathrm{O}), 1577,1450,1383$, $751 ;{ }^{1} \mathrm{H}$ NMR $\left(300 \mathrm{MHz}, \mathrm{CDCl}_{3}\right) \delta 0.65-0.80\left(\mathrm{~m}, 1 \mathrm{H}\right.$, diastereotopic $\left.-\mathrm{CH}_{2}-\right), 1.60-1.82(\mathrm{~m}, 4 \mathrm{H}$, diastereotopic $\left.-\mathrm{CH}_{2}-\right), 2.93-2.97\left(\mathrm{~m}, 2 \mathrm{H}\right.$, diastereotopic $\left.-\mathrm{CH}_{2}-\right), 2.99(\mathrm{~s}, 3 \mathrm{H}, \mathrm{MeN}), 3.15(\mathrm{~s}, 3 \mathrm{H}$, $\mathrm{MeN}), 3.71\left(\mathrm{t}, J=10.8 \mathrm{~Hz}, 1 \mathrm{H}\right.$, diastereotopic $\left.-\mathrm{CH}_{2^{-}}\right), 3.90(\mathrm{dd}, J=10.8 \mathrm{~Hz}, J \approx 3.5 \mathrm{~Hz}, 1 \mathrm{H}$, diastereotopic $\left.-\mathrm{CH}_{2}-\right), 4.43$ (s, $1 \mathrm{H},-\mathrm{CH}$-ph benzylic proton), 7.35-7.49 (m, 10H, ar.); ${ }^{13} \mathrm{C} \mathrm{NMR}$ $\left(75 \mathrm{MHz}, \mathrm{CDCl}_{3}\right) \delta 20.5\left(\mathrm{C}_{7}\right), 23.0\left(\mathrm{C}_{7}\right), 25.1\left(\mathrm{C}_{6}\right.$ or $\left.\mathrm{C}_{8}\right), 26.5\left(\mathrm{C}_{6}\right.$ or $\left.\mathrm{C}_{8}\right), 28.06\left(\mathrm{NCH}_{3}\right), 28.11$ $\left(\mathrm{NCH}_{3}\right), 28.5\left(\mathrm{C}_{2}\right.$ or $\left.\mathrm{C}_{4}\right), 35.6\left(\mathrm{C}_{2}\right.$ or $\left.\mathrm{C}_{4}\right), 40.8\left(\mathrm{C}_{1}\right.$ or $\left.\mathrm{C}_{5}\right), 49.2\left(\mathrm{C}_{1}\right.$ or $\left.\mathrm{C}_{5}\right), 51.1\left(\mathrm{C}_{3}\right.$ or $\left.\mathrm{C}_{5}\right), 51.3$ $\left(\mathrm{C}_{3}\right.$ or $\mathrm{C}_{5^{\prime}}$ ), 128.2 (C-ar.), 128.4 (C-ar.), 128.6 (C-ar.), 128.8 (C-ar.), 130.3 (C-ar.), 130.4 (C-ar.), $136.2\left(C_{i p s o}\right.$-ar. $), 137.0\left(C_{i p s o}\right.$-ar. $), 151.1\left(C_{2^{\prime}}\right), 168.1\left(C_{2^{\prime}}\right), 168.4\left(C_{4^{\prime}}\right.$ or $\left.C_{6^{\prime}}\right), 190.4\left(C_{4^{\prime}}\right.$ or $\left.C_{6^{\prime}}\right)$, $215.0\left(\mathrm{C}_{9}\right)$.

2,4-Bis(4-methoxyphenyl)-1',3'-dimethyl-1'H-spiro[bicyclo[3.3.1]nonane-3,5'-pyrimidine]$2^{\prime}, 4^{\prime}, 6^{\prime}, 9\left(3^{\prime} H\right)$-tetraone $\left(\mathbf{7 g} \boldsymbol{b}^{\prime} \boldsymbol{b}^{\prime \prime}\right)$. Yellow crystalline solid; m.p. $130-132{ }^{\circ} \mathrm{C}$ (decomps.); IR $\left(\mathrm{KBr}, \mathrm{cm}^{-1}\right) 3036$ (ar.), 3000 (ar.), 2957 (aliph.), 2935 (aliph.), 1715 (C=O), 1676 (C=O), 1609, $1512,1253,1180,1032,833,753 ;{ }^{1} \mathrm{H}$ NMR $\left(300 \mathrm{MHz}, \mathrm{CDCl}_{3}\right) \delta 1.80$ (m, 3H, diastereotopic $\left.-\mathrm{CH}_{2}-\right), 2.91\left(\mathrm{~m}, 4 \mathrm{H}\right.$, diastereotopic $\left.-\mathrm{CH}_{2}-\right), 3.14-3.35\left(\mathrm{~m}, 1 \mathrm{H}\right.$, diastereotopic $\left.-\mathrm{CH}_{2}-\right), 3.39$ (s, $3 \mathrm{H}, \mathrm{MeN}), 3.41(\mathrm{~s}, 3 \mathrm{H}, \mathrm{MeN}), 3.85(\mathrm{~s}, 6 \mathrm{H}, 2 \mathrm{MeO}), 3.68-3.91(\mathrm{~m}, 1 \mathrm{H},-\mathrm{CH}-\mathrm{ph}$ benzylic proton), 7.44 (d, $J=7.2 \mathrm{~Hz}, 4 \mathrm{H}$, ar.), 6.93 (d, $J=7.2 \mathrm{~Hz}, 4 \mathrm{H}$, ar.) ${ }^{13} \mathrm{C}_{\mathrm{NMR}}\left(75 \mathrm{MHz}, \mathrm{CDCl}_{3}\right) \delta 27.9$ $\left(\mathrm{C}_{7}\right), 28.4\left(\mathrm{C}_{6}\right.$ or $\left.\mathrm{C}_{8}\right), 41.0\left(\mathrm{NCH}_{3}\right), 43.2\left(\mathrm{NCH}_{3}\right), 49.7\left(\mathrm{C}_{2}\right.$ or $\left.\mathrm{C}_{4}\right), 55.2\left(\mathrm{C}_{1}\right.$ or $\left.\mathrm{C}_{5}\right), 55.4\left(\mathrm{OCH}_{3}\right)$, $61.3\left(\mathrm{C}_{3}\right.$ or $\left.\mathrm{C}_{5}\right), 114.1$ (C-ar.), 128.6 (C-ar.), $142.7\left(\mathrm{C}_{i p s o}\right.$-ar.), $149.8\left(\mathrm{C}_{2}\right), 159.4\left(\mathrm{C}-\mathrm{OCH}_{3}\right)$, $169.2\left(\mathrm{C}_{4^{\prime}}\right.$ or $\left.\mathrm{C}_{6^{\prime}}\right), 170.9\left(\mathrm{C}_{4^{\prime}}\right.$ or $\left.\mathrm{C}_{6^{\prime}}\right), 208.4\left(\mathrm{C}_{9}\right)$. 


\section{RESULTS AND DISCUSSION}

This article describes the crossed-aldol condensation reaction of aromatic aldehydes (1a-k) with

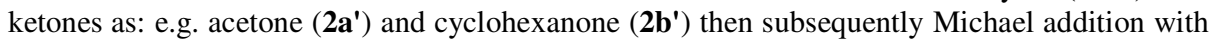
(thio)barbituric acids (4a"-4d") was afforded a new class of spiro compounds 7,11-diaryl-2,4diazaspiro[5.5]undecane-1,3,5,9-tetraones (7) (Scheme 1).

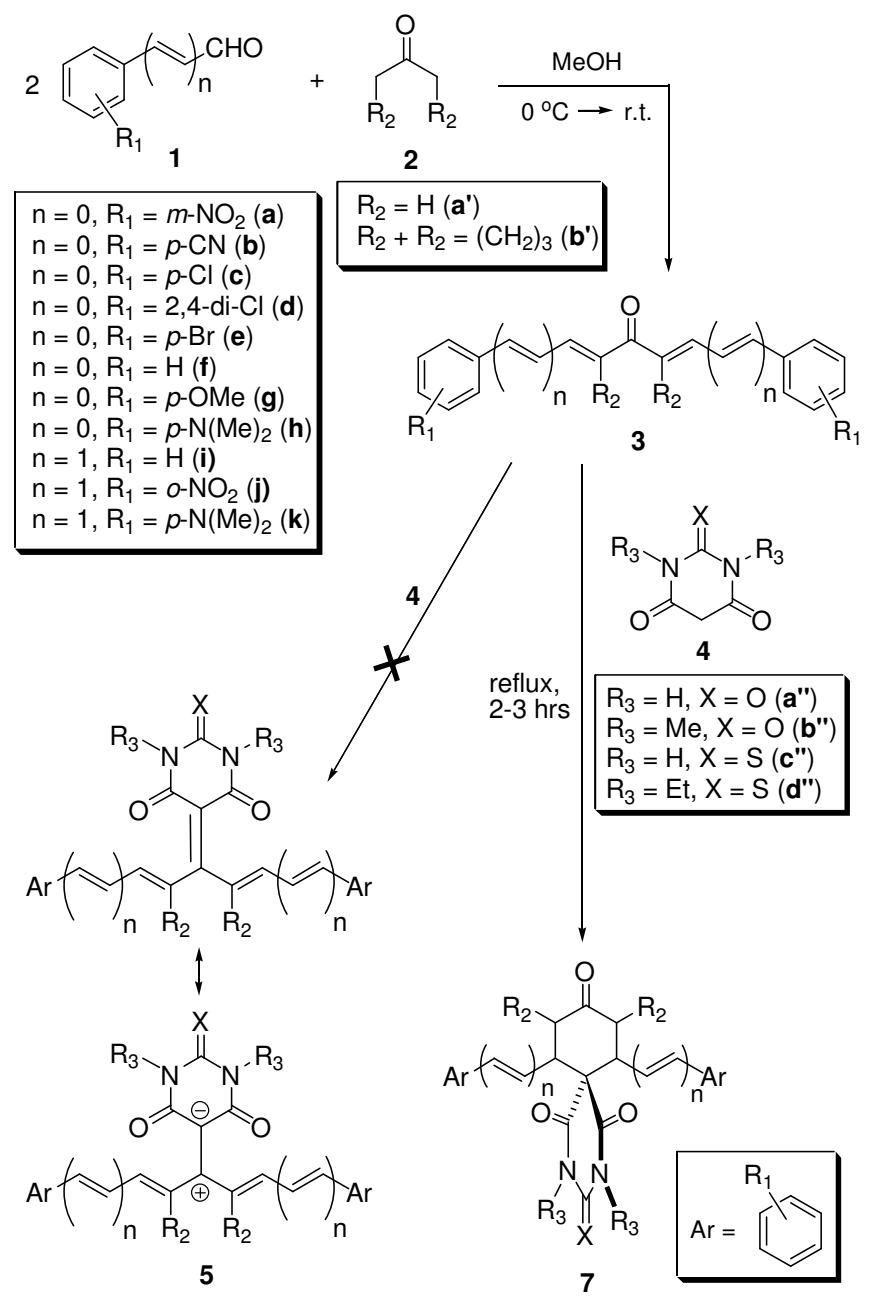

Scheme 1. Crossed-aldol condensation reaction of aromatic aldehydes (1a-k) with ketones (2a' and $\mathbf{2} \mathbf{b}^{\prime}$ ) and subsequently reaction with (thio)barbituric acids (4a"-4d") for the synthesis of 7 .

The reaction of aldehydes $\mathbf{1 a - k}$ with acetone $\mathbf{2} \mathbf{a}^{\prime}$ and cyclohexanone $\mathbf{2} \mathbf{b}^{\prime}$ leads to crossconjugated aldol condensation products, $(1 E, 4 E)$-1,5-diarylpenta-1,4-dien-3-ones (3aa'-3ha'), (2E,6E)-2,6-dibenzylidenecyclohexanones (3ab'-3hb'), (1E,3E,6E,8E)-1,9-diarylnona-1,3,6,8- 
tetraen-5-ones (3ia'-3ka') and (2E,6E)-2,6-bis $((E)$-3-phenylallylidene)cyclohexanones (3ib'3kb') (Scheme 1). The reaction between these compounds (3aa'-3ka' through 3ab'-3kb') with 4a"'-4d" were afforded spiro compounds 7. Expectedly, no symmetric zwitterionic type dyes based on (thio)barbituric acids (5) were obtained. Although, it has been reported the synthesis of zwitterionic type dyes of barbituric acids (14-16) from the reaction of barbituric acids with tropolone (11) [47], 4,9-methano[11] annulenone (12) [48] and with 7H-benzo[7]annulen-7-one (13) [49], respectively (Figure 1).

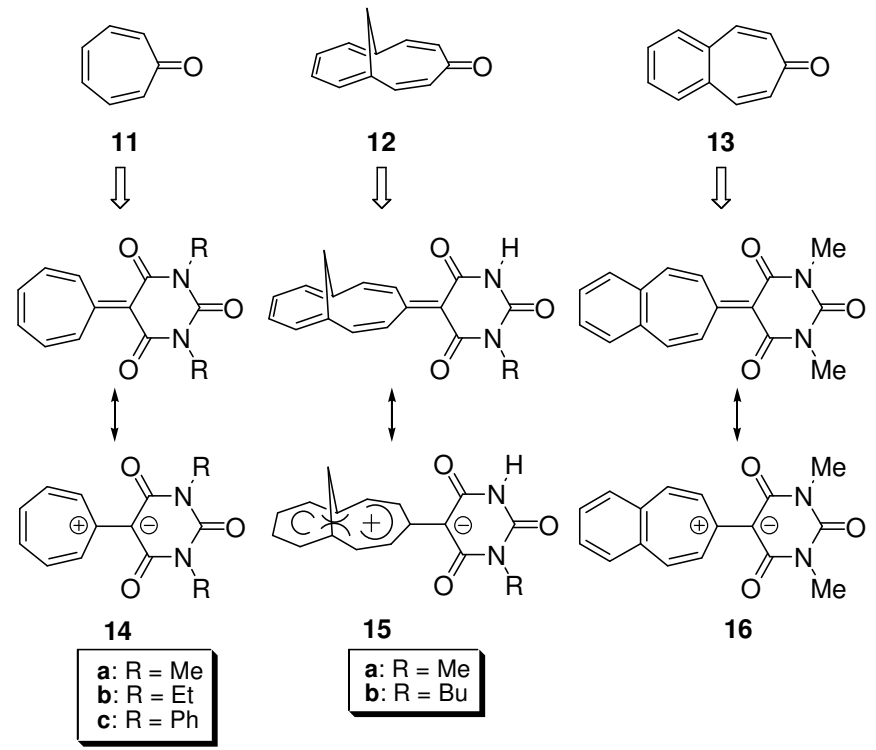

Figure 1. Structures of some zwitterionic type dyes based on barbituric acids [47-49].

Representatively, the mechanism for the formation of $\mathbf{7} \mathbf{f a}^{\prime} \mathbf{b}^{\prime \prime}$ and/or $\mathbf{7} \mathbf{f} \mathbf{b}^{\prime} \mathbf{b}^{\prime \prime}$ is shown in Scheme 2. The nucleophilic attack of 1,3-dimethyl barbituric acid $4 \mathbf{b}^{\prime \prime}$ to $\beta$-position of 3fa' and/or 3f'b' as an $\alpha, \beta$-unsaturated carbonyl compound (intermolecular Michael addition) $[55,56]$

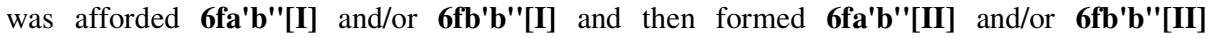
intermediates. The free-rotation around carbon-carbon single bond in $\mathbf{6} \mathbf{f a}^{\prime} \mathbf{b} \mathbf{b}^{\prime}[\mathbf{I I}]$ intermediate facilitates the second intramolecular Michael addition in the form of $\mathbf{6} \mathbf{f a} \mathbf{a}^{\prime} \mathbf{b}$ "[III] intermediate. Therefore, intramolecular Michael addition of carbon atom ( $C$-attack) in $\mathbf{6} \mathbf{f a}^{\prime} \mathbf{b}$ "'[III] intermediate afforded 2,4-dimethyl-7,11-diphenyl-2,4-diazaspiro[5.5] undecane-1,3,5,9-tetraone (7fa' $\left.\mathbf{b}^{\prime \prime}\right)$. In comparison, representatively, the reactivity of $\mathbf{3 f} \mathbf{b}^{\prime}$ is lower than that of $\mathbf{3 f a}$ ' in the reaction with $\mathbf{4} \mathbf{a}^{\prime \prime}-\mathbf{4 d}$ " due to high rigidity and rotational barrier on $\mathbf{6 f b} \mathbf{b}^{\prime} \mathbf{b}^{\prime}[\mathbf{I I}]$ than to 6fa'b' '[II] intermediate (Scheme 2). For this reason, the reaction of $\mathbf{3 f b}$ ' with $\mathbf{4} \mathbf{a}^{\prime \prime}-\mathbf{4} \mathbf{d}$ " needs to higher temperature and reaction time to be prolonged (see experimental section).

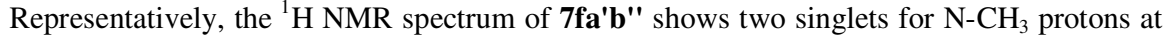
$\delta 2.88$ and $3.03 \mathrm{ppm}$, two doublet of doublets at $\delta 2.65$ and $4.05 \mathrm{ppm}$ for two diastereotopic methylene protons, a triplet at $\delta 3.75 \mathrm{ppm}$ (essentially, it is doublet of doublets) for benzylic proton on chiral stereogenic center and a multiplet at $\delta 7.07-7.28 \mathrm{ppm}$ for aromatic phenyl protons. ${ }^{13} \mathrm{C}$ NMR spectrum of this compound shows thirteen distinct peaks. One of the carbonyl peaks was extremely shifted to low field at $\delta 200.18 \mathrm{ppm}$ that confirms the formation of saturated ketone (substituted cyclohexanone). The IR spectrum of this compound shows the $\mathrm{C}=\mathrm{O}$ frequencies at 1717 and $1675 \mathrm{~cm}^{-1}$ that corresponds to carbonyl groups of spiro 
cyclohexanone and barbituric acid ring moieties, respectively. The UV-Visible spectra of some cross-conjugated dyes such as 3fa', 3ca' and some corresponding spiro compounds $\mathbf{7} \mathbf{f a}^{\prime} \mathbf{b}^{\prime}$ ', 7fa'd" and 7ca'b" is shown in Figure 2. Representatively, the 3fa' shows two absorption bonds at 320 and $384 \mathrm{~nm}$ that corresponds to $\pi \rightarrow \pi^{*}$ (K-band) and weak $\mathrm{n} \rightarrow \pi^{*}$ (R-band), respectively.

2<smiles>O=Cc1ccccc1</smiles>

$1 f$<smiles>O=C1CCCCC1</smiles>

$2 a^{\prime} \& 2 b^{\prime}$
(2)
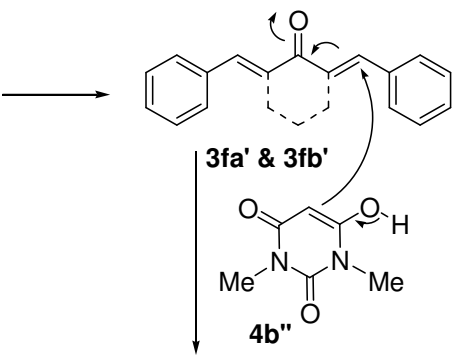<smiles>CN1C(=O)C(=Cc2ccccc2)C(=O)C(C(c2ccccc2)C2CCCCC2=O)C1=O</smiles>

4 6fa'b"[II] \& 6fb'b"[II]

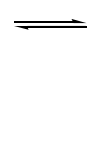

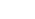<smiles>CN1C(=O)C(C(C2=C(O)C(=Cc3ccccc3)CCC2)c2ccccc2)C(=O)N(C)C1=O</smiles>

6fa'b"[l] \& 6fb'b"[l]<smiles>Cn1c(=O)c(-c2ccccc2)c(C(c2ccccc2)c2ccccc2)c(=O)n1C</smiles>

6fa'b"[III] \& 6fb'b"[III]

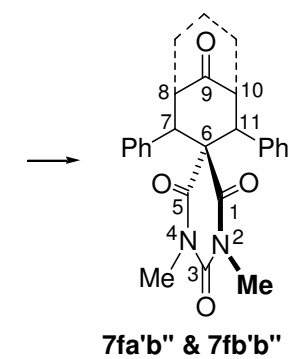

Scheme 2. Representatively, proposed mechanism for the formation of $\mathbf{7 f a} \mathbf{\prime}^{\prime} \mathbf{b}^{\prime \prime}$ and/or $\mathbf{7} \mathbf{f b}^{\prime} \mathbf{b}^{\prime \prime}$ (the rotation mark $\left(C^{2}\right)$ is only shown for $\mathbf{6} \mathbf{f a}^{\prime} \mathbf{b}^{\prime}[\mathbf{I I}]$ intermediate).

In contrast, in $\mathbf{7} \mathbf{f a}^{\prime} \mathbf{b}^{\prime \prime}$, the allowed $\pi \rightarrow \pi^{*}$ transition was disappeared and the only forbidden weak $\mathrm{n} \rightarrow \pi^{*}$ band at $304 \mathrm{~nm}$ was observed (see experimental section). Obviously, in dyes 3fa' and 3ca' the K-band undergoes a small bathochromic shift because of the enone conjugation. Instead, in 7fa'b" and 7fa'd" the K-bank hypsochromically shifted. These data also confirm the formation of the saturated cyclohexanone (decreasing the conjugated system) in $\mathbf{7} \mathbf{f a}^{\prime} \mathbf{b}$ " and 7fa'd" as representative. A small absorption at $402 \mathrm{~nm}$ for $\mathbf{7 f a} \mathbf{d}^{\prime \prime}$ ', corresponds to $\mathrm{n} \rightarrow \pi^{*}$ band of thiocarbonyl group (Figure 2).

The proposed mass fragmentation of $\mathbf{7 f a} \mathbf{a}^{\prime \prime}$ and $\mathbf{7} \mathbf{f a}^{\prime} \mathbf{b}^{\prime \prime}$ is shown in Scheme 3. The molecular ion peaks of 7fa'a" and 7fa'b" are shown at $\mathrm{m} / \mathrm{z}, 362(65 \%)$ and $390(45 \%$ abundance), respectively. Representatively, the ion molecular of $7 \mathbf{f a}^{\prime} \mathbf{a}^{\prime \prime}$ cleaved to styrene at $\mathrm{m} / \mathrm{z} 104$ (100\% abundance) as a base peak and ion radical fragment of 3-phenyl-6,8diazaspiro[3.5]nonane-1,5,7,9-tetraone (A) at $m / z, 258$ (25\%). This later fragment (A) converted to ion radical fragment of 1-phenyl-5,7-diazaspiro[2.5] octane-4,6,8-trione (C) at $\mathrm{m} / z, 230(90 \%)$ 


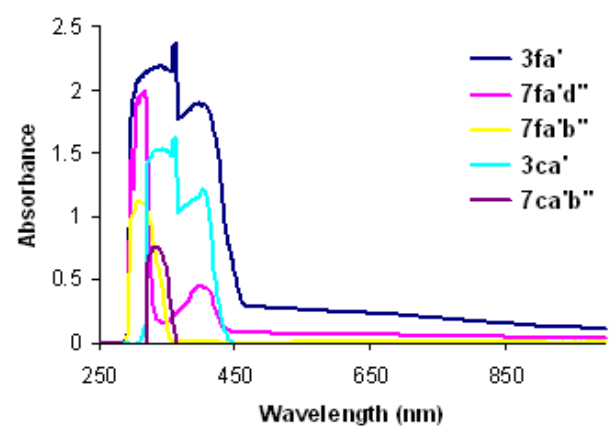

Figure 2. UV-Visible spectra of some selected dyes and corresponding spiro compounds.
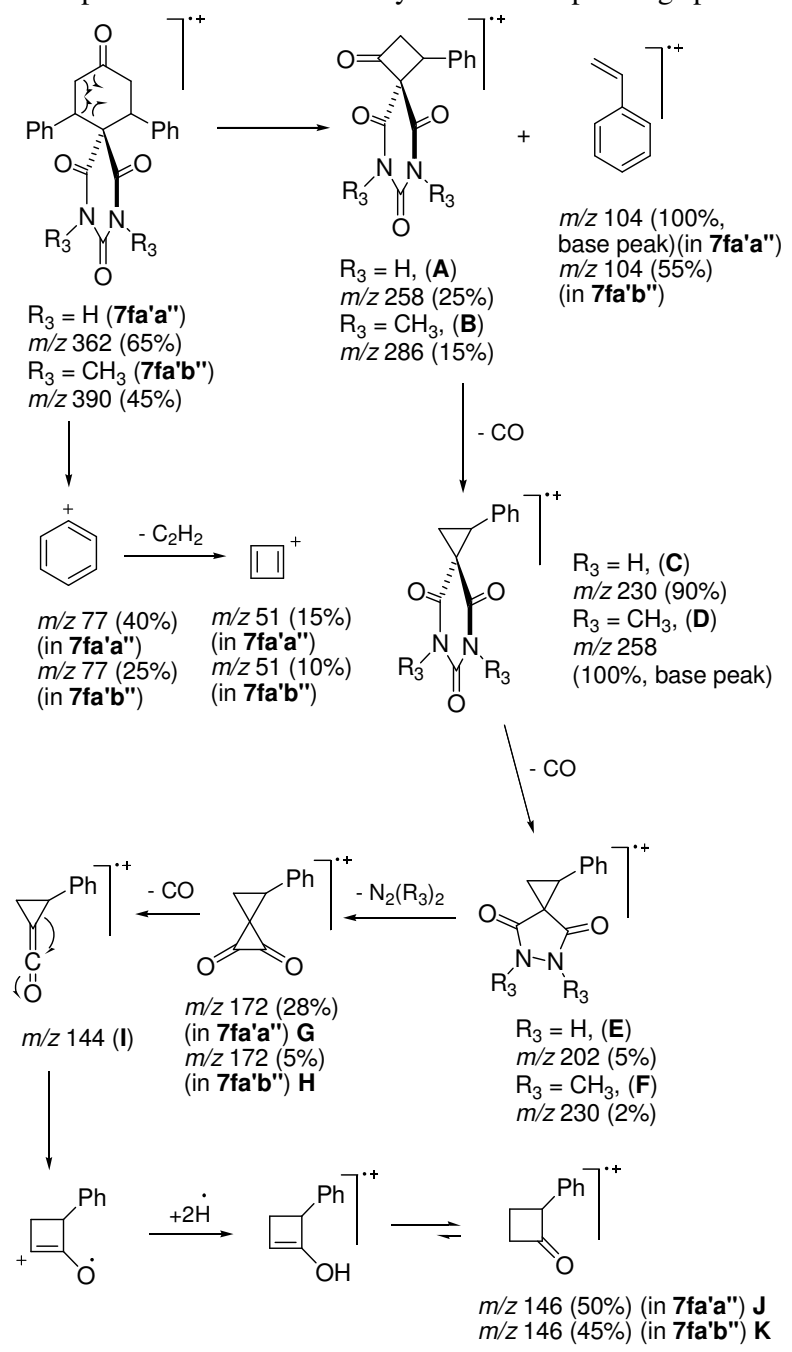

Scheme 3. Representatively, proposed mass fragmentation of 7fa'a" and 7fa'b".

Bull. Chem. Soc. Ethiop. 2014, 28(3) 
by loss of carbon monoxide (CO). The twice loss of $\mathrm{CO}$ from this fragment generated the fragment of $m / z 202(\mathbf{E})$, then the loss of diazene $\left(\mathrm{N}_{2} \mathrm{H}_{2}\right)$ obtained 4-phenylspiro[2.2]pentane1,2-dione ion radical (G) at $\mathrm{m} / z \quad 172 \quad(28 \%)$. The ion radical fragment of (2phenylcyclopropylidene)methanone (I) at $\mathrm{m} / z, 144$ generated from this fragment by loss of CO. The intramolecular rearrangement of phenylcyclopropylidene)methanone ion radical then capturing two hydrogen radicals and finally tautomerized to 2-phenylcyclobutanone ion radical (J) at $\mathrm{m} / \mathrm{z}, 146(50 \%)$ (Scheme 3). Similarly, the same fragmentation of $\mathbf{7} \mathbf{f a}^{\prime} \mathbf{b}$ " is also shown in Scheme 3 .

(a)

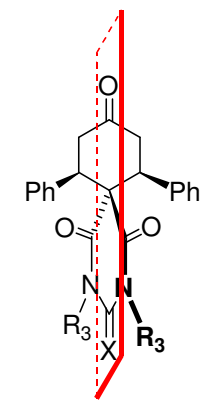

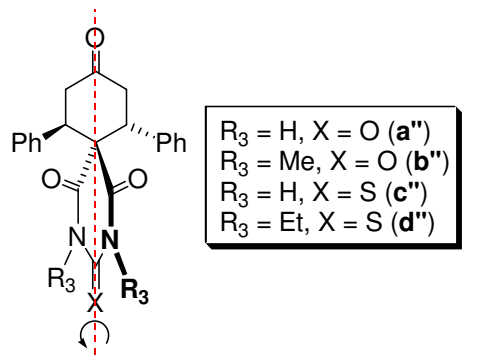

Plane of symmetry Axis of symmetry $\left(C_{2}\right)$

A

7fa'a"-d"

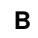

(b)

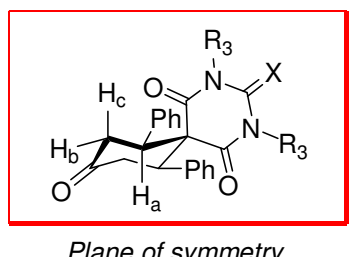

Plane of symmetry

$7 f a ' a "-d "$

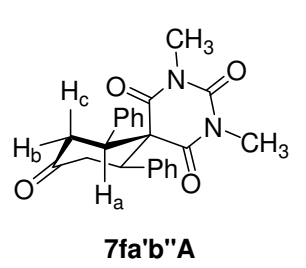

$$
\begin{array}{|lll|}
\hline \mathrm{H}_{\mathrm{a}}: 4.04 \mathrm{ppm} & \mathrm{H}_{\mathrm{b}}: 2.64 \mathrm{ppm} & \mathrm{H}_{\mathrm{c}}: 3.75 \mathrm{ppm} \\
\mathrm{J}_{\mathrm{ac}}=14.4 \mathrm{~Hz} & J_{\mathrm{bc}}=14.7 \mathrm{~Hz} & J_{\mathrm{ca}}=14.4 \mathrm{~Hz} \\
\mathrm{Jab}_{\mathrm{ab}}=4.2 \mathrm{~Hz} & J_{\mathrm{ba}}=4.2 \mathrm{~Hz} & J_{\mathrm{cb}}=14.7 \mathrm{~Hz} \\
\hline
\end{array}
$$

Figure 3. Representatively, possible structural conformation of 7fa'a"'-d" (a), the observed plane of symmetry in 7fa'a"'-d" (b) and the chemical shifts and coupling constants of assigned protons in $\mathbf{7 f a} \mathbf{\prime b}^{\prime \prime} \mathbf{A}$ as representative (c).

Two possible conformers (also diastereomers) of $\mathbf{7 f a} \mathbf{a}^{\prime \prime}$-'d" is shown in Figure 3a. Representatively, compound 7fa'b" can be serves as plane of symmetry (7fa'b"'A form) and 
axis of symmetry, $C_{2}$ (7fa'b"'B form) (Figure 3a). The Newman projection and observed structural conformers of $\mathbf{7} \mathbf{f a}^{\prime} \mathbf{a}^{\prime \prime}-\mathbf{d}$ "' is shown in Figure 3b. Representatively, the predominant conformer and coupling constants $(J)$ of $\mathbf{7 f a} \mathbf{\prime}^{\prime} \mathbf{b}^{\prime \prime} \mathbf{A}$ is also shown in Figure $3 \mathrm{c}$. The dependence of vicinal coupling constants on the dihedral angle, $\varphi$, based on Karplus curve - is described [57, 58]. For instance, in $\mathbf{7} \mathbf{f a}^{\prime} \mathbf{b}^{\prime \prime} \mathbf{A}$ form, for $\mathrm{H}_{\mathrm{a}}$ at $\delta 4.04 \mathrm{ppm}$, the dihedral angle of $\varphi_{\mathrm{ac}}$ and $\varphi_{\mathrm{ab}}$ are equals to $180^{\circ}$ and $60^{\circ}$, respectively. The corresponding $J$-values were obtained 14.4 and 4.2 $\mathrm{Hz}$, respectively. For $\mathrm{H}_{\mathrm{b}}$ at $\delta 2.64 \mathrm{ppm}$, the $J$-values were obtained $14.7 \mathrm{~Hz}$ for two geminal protons $\mathrm{H}_{\mathrm{b}}$ and $\mathrm{H}_{\mathrm{c}}$ and $4.2 \mathrm{~Hz}$ for vicinal $\mathrm{H}_{\mathrm{b}}$ and $\mathrm{H}_{\mathrm{a}}$, respectively $\left(\varphi_{\mathrm{ab}}=60^{\circ}\right)$. For $\mathrm{H}_{\mathrm{c}}$, the $J$ values were obtained 14.4 and $14.7 \mathrm{~Hz}$ for vicinal $\left(J_{\mathrm{ca}}\right)$ and geminal protons $\left(J_{\mathrm{cb}}\right)$, respectively.
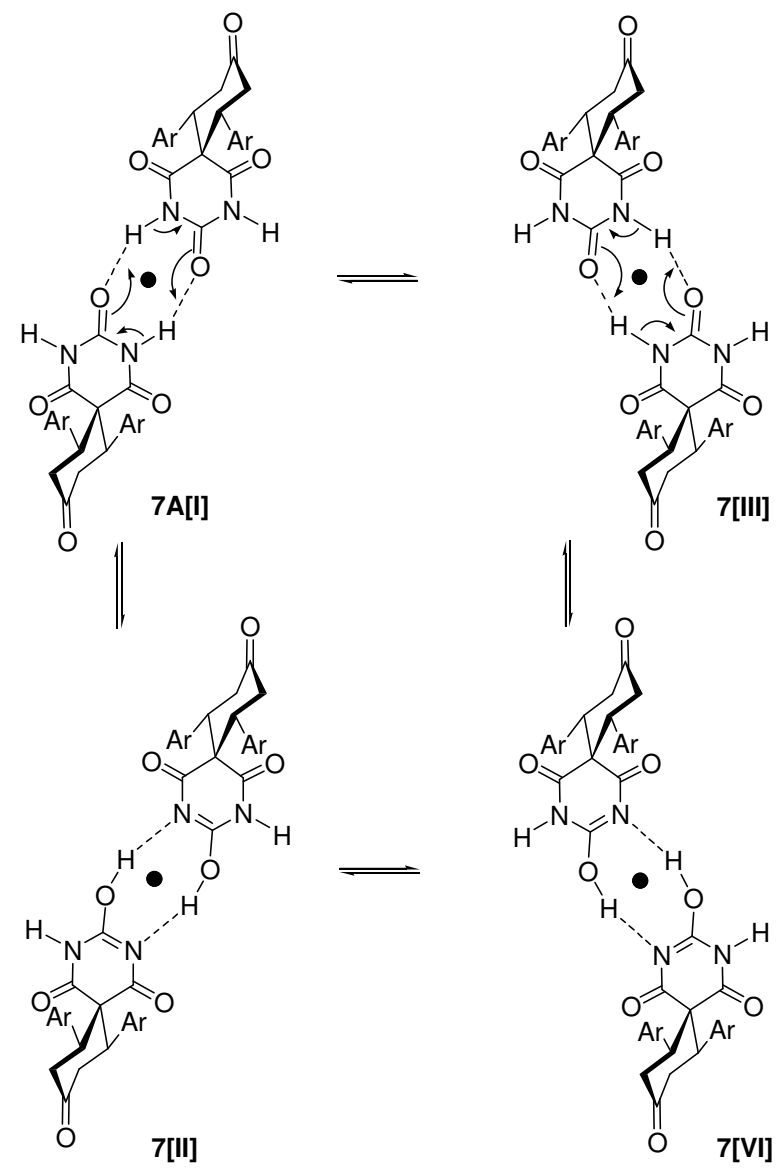

$\mathrm{Ar}=p-\mathrm{Cl}-\mathrm{C}_{6} \mathrm{H}_{4}$ (7ca'a"), $p-\mathrm{Br}^{-} \mathrm{C}_{6} \mathrm{H}_{4}$ (7ea'a"), p-MeO- $\mathrm{C}_{6} \mathrm{H}_{4}$ (7ga'a")

Scheme 4. Representatively, two intermolecular hydrogen bonds and tautomeric forms of 7ca'a", 7ea'a" and 7ga'a".

These observations indicated that the bulky phenyl substituents lie in equatorial positions in 7 and cyclohexanone ring moiety has chair conformer (Figure $3 b, c$ ). In addition, the ${ }^{1} \mathrm{H},{ }^{13} \mathrm{C}$ 
NMR, IR, mass fragmentation data and conformational analysis confirms the assigned structure of $7 \mathbf{f a}^{\prime} \mathbf{b}$ "A (including a plane of symmetry, $\sigma$ ). These data indicated that representatively, 7fa'a"-7fa'd" have plane of symmetry and are in 7fa'a"-7fa'd"A forms (Figure 3). No spiro barbiturates $7 \mathbf{f a} \mathbf{a}^{\prime}$ "-7fa'd"'B forms consists of axis of symmetry $\left(C_{2}\right)$ were observed based on NMR investigations. The chemical shifts of $\mathrm{N}-\mathrm{R}_{3}$ protons to be equivalent in (thio)barbituric acid ring moiety if the molecule have axis of symmetry $\left(C_{2}\right)$.

Another most interesting phenomenon in the spiro-barbiturate derivatives of $\mathbf{4 a "}$ is the equivalence of the chemical shifts of two NH protons that appeared as an extremely broad singlet. This phenomenon presumably corresponds to the intermolecular hydrogen bond of $\mathrm{NH}$ protons in the molecule with oxygen atom on amidic carbonyl group of other molecule one (Scheme 4). Instead, this equivalency of two NH protons was not shown in the spirothiobarbiturate derivatives derived from $\mathbf{4} \mathbf{c}^{\prime \prime}$ (Figure 4). Representatively, the equivalency of the NH protons of 7ca'a" and 7ea'a" are shown and comparised with the NH protons of 7ca'c" and 7ea'c"' (thiobarbituric acid derivatives) in Figure 4.

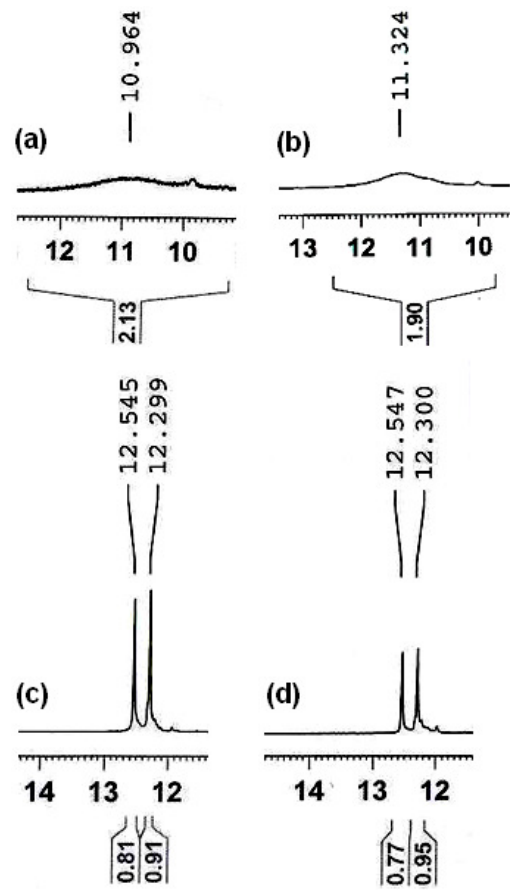

Figure 4. Representatively, expanded ${ }^{1}$ H NMR spectra of 7ca'a" (a), 7ea'a" (b), 7ca'c" (c) and 7ea'c" (d) at NH region.

We have developed the reaction of 1a"-1d" with (2E,6E)-2,6-diarylidenecyclohexanones $\left(3 \mathbf{a b}^{\prime}-\mathbf{3 k b} \mathbf{k}^{\prime}\right)$ in ethanol in both basic $\left(\mathrm{Et}_{3} \mathrm{~N}\right)$ and acidic condition $(\mathrm{AcOH})$. Similar to reaction of acetone $\mathbf{2} \mathbf{a}^{\prime}$, the reaction between cyclohexanone $\mathbf{2} \mathbf{b}^{\prime}$ with aromatic aldehydes $\mathbf{1 a - 1} \mathbf{k}$, were afforded 3ab'-3kb'. The reaction of these dyes with $\mathbf{4 a} \mathbf{a}^{\prime \prime}-\mathbf{4 d}$ " gave $\mathbf{7} \mathbf{a b}^{\prime} \mathbf{a}^{\prime \prime}-\mathbf{7 k b} \mathbf{k b}^{\prime \prime}$ through 7ab'd"-7kb'd" (Scheme 1). Representatively, the reaction of benzaldehyde 1f with cyclohexanone $\mathbf{2} \mathbf{b}^{\prime}$ afforded $\mathbf{3 f} \mathbf{b}^{\prime}$ then its reaction with DMBA $\mathbf{4} \mathbf{b}^{\prime \prime}$ gave 1',3'-dimethyl-2,4diphenyl-1' $H$-spiro[bicyclo[3.3.1]nonane-3,5'-pyrimidine]-2',4',6',9(3'H)-tetraone (7ffb'b'"). No 
symmetrical crossed-conjugated type dye 5-((2E,6E)-2,6-dibenzylidenecyclohexylidene)-1,3dimethylpyrimidine-2,4,6(1H,3H,5H)-trione (5fb'b") and/or its zwitterionic form was observed (Scheme 5).

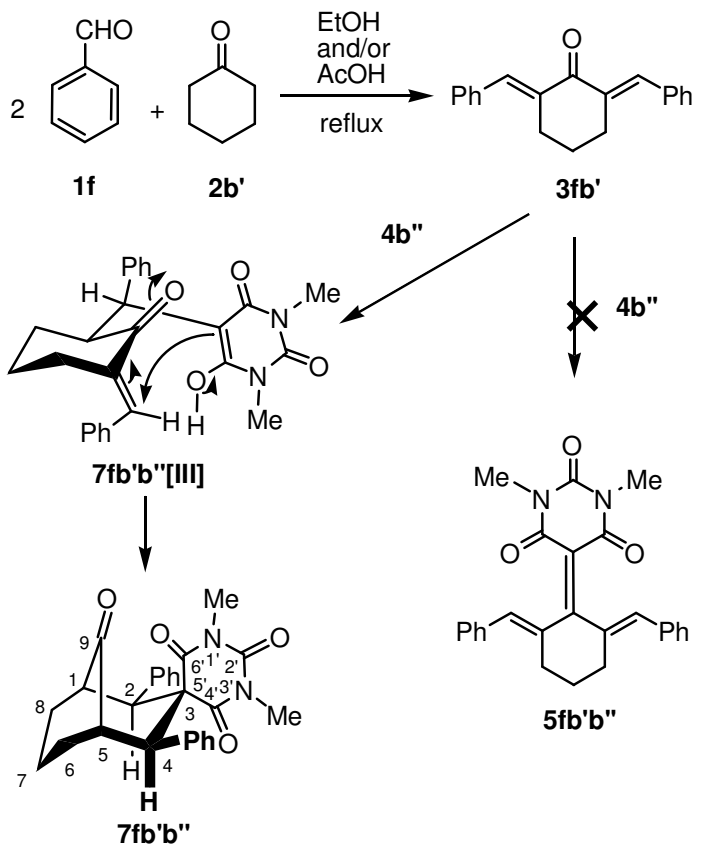

Scheme 5. Representatively, the reaction of benzaldehyde (1f) with cyclohexanone (2b') formed 3fb' and then subsequently reaction mechanism with 1,3-dimethyl barbituric acid (4b") formed $7 \mathbf{f} \mathbf{b}^{\prime} \mathbf{b} "$.

Representatively, the ${ }^{1} \mathrm{H}$ NMR spectrum of $\mathbf{7} \mathbf{f b}^{\prime} \mathbf{b}$ " shows multiplets at $\delta 0.65-0.80 \mathrm{ppm}$ $(1 \mathrm{H})$, at $\delta 1.60-1.82 \mathrm{ppm}(4 \mathrm{H})$ and at $\delta 2.93-2.97 \mathrm{ppm}(2 \mathrm{H})$ for diastereotopic protons on spiro bicyclo scaffold. ${ }^{1} \mathrm{H}$ NMR spectrum of this compound also show two singlets for $\mathrm{N}-\mathrm{CH}_{3}$ protons at $\delta 2.99(3 \mathrm{H})$ and $3.15 \mathrm{ppm}(3 \mathrm{H})$, a triplet at $\delta 3.71 \mathrm{ppm}(1 \mathrm{H}, J=10.8 \mathrm{~Hz})$, a doublet of doublets at $\delta 3.90 \mathrm{ppm}(1 \mathrm{H}, J=10.8 \mathrm{~Hz}, J \approx 3.5 \mathrm{~Hz})$, a singlet at $\delta 4.43 \mathrm{ppm}(1 \mathrm{H})$ and a multiplet at $\delta 7.35-7.49 \mathrm{ppm}$ for aromatic phenyl protons. Obviously, the ${ }^{13} \mathrm{C}$ NMR spectrum of this compound shows fifteen distinct peaks. One of the carbonyl peaks was extremely shifted to low field at $\delta 215.0 \mathrm{ppm}$ that confirms the formation of saturated ketone (substituted spiro bicyclo[3.3.1]nonan-9-one scaffold). The IR spectrum of this compound shows the $\mathrm{C}=\mathrm{O}$ frequencies at 1743 and $1672 \mathrm{~cm}^{-1}$ that corresponds to carbonyl groups of spiro bicyclo[3.3.1]nonan-9-one moiety in 7fb'b". All structures of 7ab'a"'-7kb'a" through 7ab'd"7kb'd" (structures derived from 3ab'-3kb') may have two diastereomers due to having two chiral stereogenic centers. For this reason, the ${ }^{1} \mathrm{H}$ and ${ }^{13} \mathrm{C}$ NMR spectra of the many of these compounds are complicated. Possible structural conformations of 7fb'a' '-d" ([I], [II] and [III] forms) are shown in Figure 5. We have adjourned the conformational studies of these compounds by means of advanced NMR techniques in the near future. 

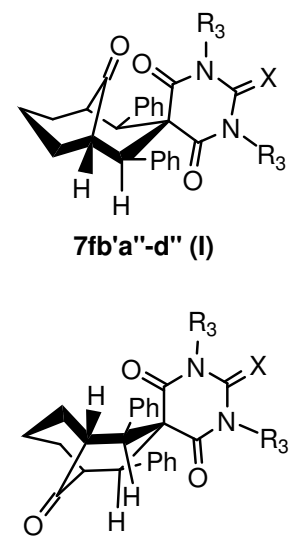

(III)

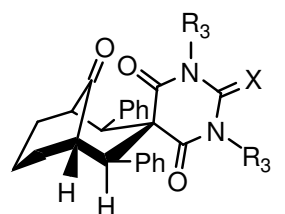

(II)

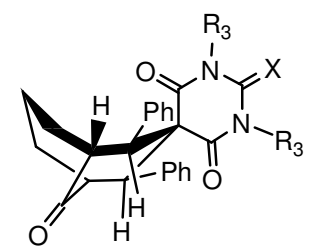

(IV)

$$
\begin{aligned}
& \mathrm{R}_{3}=\mathrm{H}, \mathrm{X}=\mathrm{O}\left(\mathbf{a}^{\prime \prime}\right) \\
& \mathrm{R}_{3}=\mathrm{Me}, \mathrm{X}=\mathrm{O}\left(\mathbf{b}^{\prime \prime}\right) \\
& \mathrm{R}_{3}=\mathrm{H}, \mathrm{X}=\mathrm{S}\left(\mathbf{c}^{\prime \prime}\right) \\
& \mathrm{R}_{3}=\mathrm{Et}, \mathrm{X}=\mathrm{S}\left(\mathbf{d}^{\prime \prime}\right)
\end{aligned}
$$

Figure 5. Representatively, possible structural conformations of 7fb'a"-d"'.

\section{$X$-Ray data for $\mathbf{7} \mathbf{c a}^{\prime} \mathbf{b}^{\prime \prime}$}

For the further study, the crystal structure and packing diagram of compound 7ca'b" are shown in Figures 6 and 7, respectively. The cyclohexanone and barbituric acid rings have chair and nearly planar conformers, respectively. The torsion angle between two phenyls and cyclohexanone rings obtained in results of 79.93 and $95.57^{\circ}$, respectively.

The summary of crystal date for 7ca'b": crystal system, space group: tetragonal, P-42 ${ }_{1} \mathbf{c}$; unit cell dimensions: $a=21.5533(5), b=21.5533(5), c=9.4674(2) \AA, \alpha=90.00, \beta=90.00, \gamma=$ 90.00, cell volume: $4398.03 \AA^{3} ; Z=8$. Single crystal of 7ca'b" was obtained as colorless crystal by slow evaporation from methanol at room temperature. For the crystal structure determination, the single-crystal of the compound 7 $\mathbf{c a}^{\prime} \mathbf{b}^{\prime \prime}$ was used for data collection on a four-circle Rigaku R-AXIS RAPID-S diffractometer (equipped with a two-dimensional area IP detector). The graphite-monochromatized Mo $\mathrm{K}_{\alpha}$ radiation $(\lambda=0.71073 \AA)$ and oscillation scans technique with $\Delta \omega=5^{\circ}$ for one image were used for data collection. The lattice parameters were determined by the least-squares methods on the basis of all reflections with $F^{2}>2 \sigma\left(\mathrm{F}^{2}\right)$. Integration of the intensities, correction for Lorentz and polarization effects and cell refinement was performed using CrystalClear (Rigaku/MSC Inc., 2005) software [59]. The structures were solved by direct methods using SHELXS-97 [60] and refined by a full-matrix least-squares procedure using the program SHELXL-97 [60]. $H$-atoms were positioned geometrically and refined using a riding model. The final difference Fourier maps showed no peaks of chemical significance. Crystallographic data were deposited in CSD under CCDC-800579 registration number and are available free of charge upon request to CCDC, 12 Union Road, Cambridge, UK (fax: +44-1223-336033, e-mail: deposit@ccdc.cam.ac.uk). 


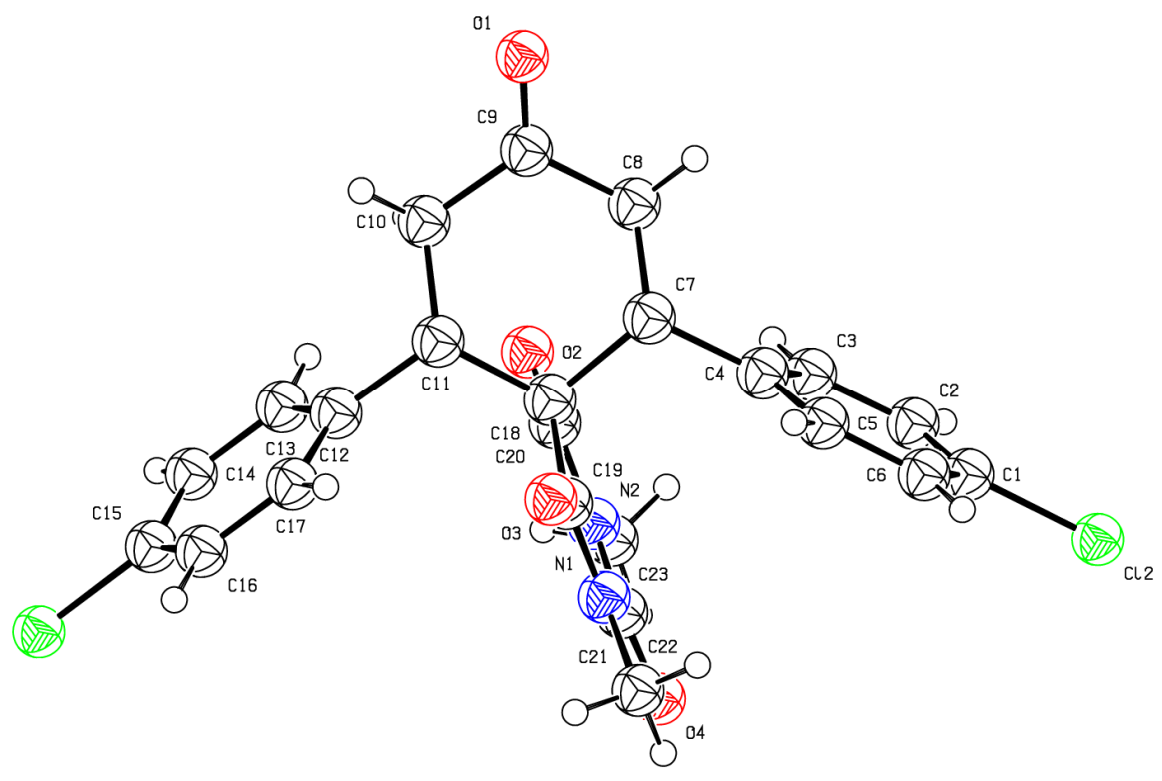

Figure 6. Crystal structure of 7ca'b".

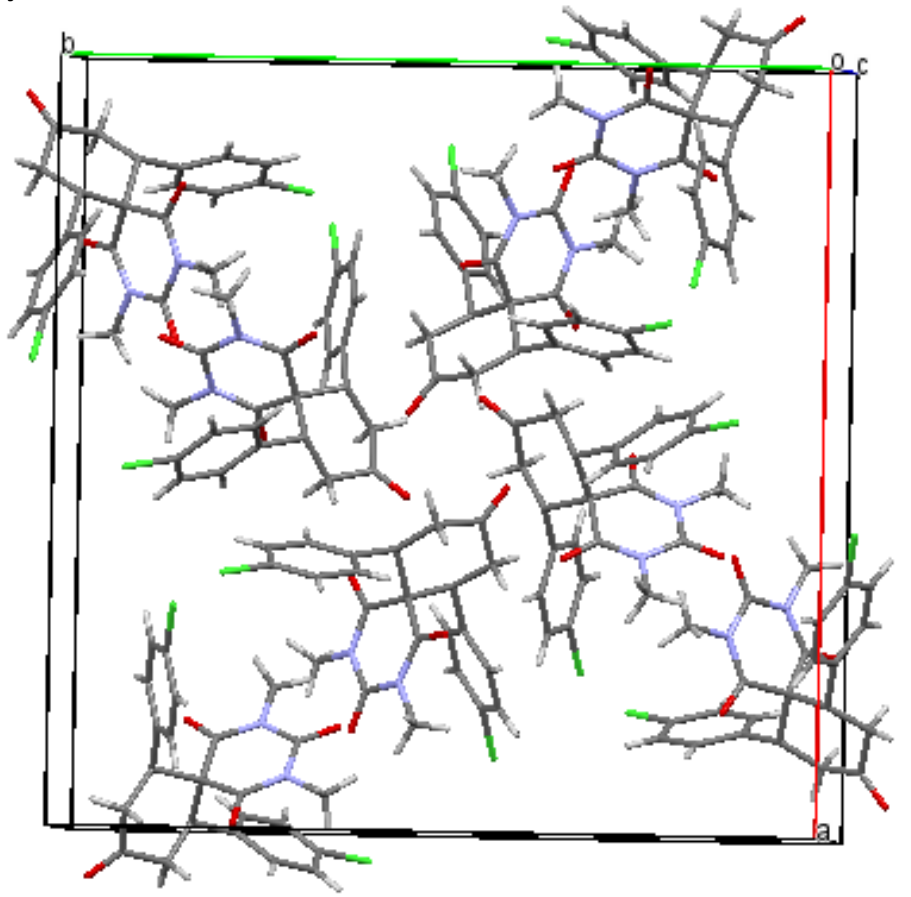

Figure 7. Crystal packing diagram of 7ca'b".

Bull. Chem. Soc. Ethiop. 2014, 28(3) 


\section{CONCLUSION}

In summary, the crossed-aldol condensation reaction of aromatic aldehydes bearing electron donating and electron withdrawing substituents with acetone and cyclohexanone afforded crossconjugated type dyes. The inter- and then intramolecular Michael addition reaction of these dyes with (thio)barbituric acids afforded new type of spiro (thio)barbiturates and bicyclo spiro (thio)barbiturates, respectively. The structures of spiro (thio)barbiturates derived from acetone revealed that the spiro cyclohexanone moiety has chair conformer and two phenyl (and/or aryl) groups on cyclohexanone lies in equatorial position. The crystal structure of the one of spiro compounds derived from acetone show that the cyclohexanone and barbituric acid rings have chair and nearly planar conformers, respectively, in solid state.

\section{ACKNOWLEDGEMENTS}

We gratefully acknowledge financial support by the Research Council of Urmia University. Authors also acknowledge to crystallography laboratory management in Atatürk University, Erzurum, Turkey.

\section{REFERENCES}

1. Deli, J.; Lorand, T.; Szabo, D.; Foldesi, A. Pharmazie 1984, 39, 539.

2. Nielsen, A.T.; Houlihan, W.J. Org. React. 1968, 16, 1.

3. Guilford, W.J.; Shaw, K.J.; Dallas, J.L.; Koovakkat, S.; Lee, W.; Liang, A.; Light, D.R.; McCarrick, M.A.; Whitlow, M.; Ye, B.; Morrissey, M.M. J. Med. Chem. 1999, 42, 5415.

4. Riadi, Y.; Mamouni, R.; Azzalou, R.; Boulahjar, R.; Abrouki, Y.; El Haddad, M.; Routier, S.; Guillaumet, G.; Lazar, S. Tetrahedron Lett. 2010, 51, 6715.

5. Artico, M.; Di Santo, R.; Costi, R.; Novellino, E.; Greco, G.; Massa, S.; Tramintano, E.; Marongiu, M.E.; De Montis, A.; La Colla, P. J. Med. Chem. 1998, 41, 3948.

6. Jia, Z.C.; Quail, J.W.; Arora, V.K.; Dimmock, J.R. Acta Crystallogr. Sect. C. 1989, 45, 1117.

7. Behera, R.K.; Behera, A.K.; Pradhan, R. Patra, M. Indian J. Chem. 2006, 45B, 933.

8. Ogawa, M.; Ishii, Y.; Nakano, T.; Irifune, S. JP 63192446, 1988; Chem. Abstr. 1988, 63, 238034.

9. Gangadhara, K.K. Polymer 1995, 36, 1903.

10. Trost, B.M.; Fleming, I. in Comprehensive Organic Synthesis, Vol. 3, Pergamon Press: Oxford, 1991, Chapter 1.4.

11. Norcross, R.D.; Paterson, I. Chem. Rev. 1995, 95, 2041.

12. Smith, M.B. March, J. Advanced Organic Chemistry, Reactions, Mechanisms, and Structure, John Wiley and Sons: New York; 2001; p 1218.

13. Hathaway, B.A. J. Chem. Educ. 1987, 64, 367.

14. Wang, J.; Kang, L.; Hu, Y.; Wie, B. Synth. Commun. 2002, 32, 1691.

15. Li, J.; Yang, W.; Chen, G.; Li, T. Synth. Commun. 2003, 33, 2619.

16. Wang, L.; Sheng, J.; Tian, H.; Han, J.; Fan, Z.; Qian, C. Synthesis 2004, 3060.

17. Sabitha, G.; Reddy, K.K.; Reddy, K.B.; Yadav, J.S. Synthesis 2004, 263.

18. Iranpoor, N.; Zeynizadeh, B.; Aghapour, A. J. Chem. Res. 1999, 554.

19. Iranpoor, N.; Kazemi, F. Tetrahedron 1998, 54, 9475.

20. Zhu, Y.; Pan, Y. Chem. Lett. 2004, 33, 668.

21. Hu, X.; Fan, X.; Zhang, X.; Wang, J. J. Chem. Res. 2004, 684.

22. Zheng, X.; Zhang, Y. Synth. Commun. 2003, 33, 161.

23. Das, B.; Thirupathi, P.; Mahender, I.; Reddy, K.R. J. Mol. Catal. A: Chem. 2006, 247, 182.

24. Bhagat, S.; Sharma, R.; Charaborti, A.K. J. Mol. Catal. A: Chem. 2006, 260, 235. 
25. Singh, N.; Pandey, J.; Yadav, A.; Chaturvedi, V.; Bhatnagar, S.; Gaikwad, A.N.; Sinha, S.K.; Kumar, A.; Shukla, P.K.; Tripathi, R.P. Eur. J. Med. Chem. 2009, 44, 1705.

26. Vashchenko, V.; Kutulya, L.; Krivoshey, A. Synthesis 2007, 2125.

27. Ryabukhin, S.V.; Plaskon, A.S.; Volochnyuk, D.M.; Pipko, S.E.; Shivanyuk, A.N.; Tolmachev, A.A. J. Comb. Chem. 2007, 9, 1073.

28. Abaee, M.S.; Mojtahedi, M.M.; Sharifi, R.; Zahedi, M.M.; Abbasi, H.; Tabar-Heidar, K. J. Iran. Chem. Soc. 2006, 3, 293.

29. Jin, T.S.; Zhao, Y.; Liu, L.B.; Li, T.S. Indian J. Chem. 2007, 45, 2229.

30. Zhang, X.; Fan, X.; Niu, H.; Wang, J. Green Chem. 2003, 5, 267.

31. Zahouily, M.; Abrouki, Y.; Rayadh, A.; Sebti, S.; Dhimane, H.; David, M. Tetrahedron Lett. 2003, 44, 2463.

32. King, S.B.; Stratford, E.S.; Craig, C.R.; Fifer, E.K. Pharm. Res. 1995, 12, 1240.

33. Fraser, W.; Suckling, C.J.; Wood, H.C.S. J. Chem. Soc. Perkin. Trans. I 1990, 11, 3137.

34. Ingle, V.N.; Gaidhane, P.K.; Hatzade, K.M.; Umare, V.D.; Taile, V.S. Int. J. Pharm. Tech. Res. 2009, 1, 605.

35. King, S.B.; Stratford, E.S.; Craig, C.R.; Fifer, E.K. Pharm. Res. 1995, 12, 1240.

36. Kesharwani, S.; Sahu, N.K.; Kohli, D.V. Pharm. Chem. J. 2009, 43, 315.

37. Al-Ashmawi, M.I.; Ghoneim, K.M.; Khalifa, M. Pharmazie 1980, 35, 591.

38. Krasnov, K.A.; Kartsev, V.G.; Khrustalev, V.N. Mendeleev Commun. 2006, 16, 52-54.

39. Elinson, M.N.; Vereshchagin, A.N.; Stepanov, N.O.; Zaimovskaya, T.A.; Merkulova, V.M.; Nikishin, G.I. Tetrahedron Lett. 2010, 51, 428.

40. Baruah, B.; Bhuyan, P.J. Tetrahedron Lett. 2009, 50, 243.

41. Elinson, M.N.; Vereshchagin, A.N.; Stepanov, N.O.; Belyakov, P.A.; Nikishin, G.I. Tetrahedron Lett. 2010, 51, 6598.

42. Noroozi Pesyan, N.; Rastgar, S.; Hosseini, Y. Acta Cryst. Sect. E 2009, 65, o1444.

43. Jalilzadeh, M.; Noroozi Pesyan, N.; Rezaee, F.; Rastgar, S.; Hosseini, Y.; Şahin, E. Mol. Divers. 2011, 15, 721.

44. Hosseini, Y.; Rastgar, S.; Heren, Z.; Büyükgüngör, O.; Noroozi Pesyan, N. J. Chin. Chem. Soc. 2011, 58, 309.

45. Ramachary, D.B.; Barbas, C.F. Org. Lett. 2005, 7, 1577.

46. Khurana, J.M.; Arora, R.; Satija, S. Heterocycles 2007, 71, 2709.

47. Naya, S.; Nitta, M. Tetrahedron 2003, 59, 3709.

48. Vogel, E. 23rd Int. Congr. Pure Appl. Chem. 1971, 1, 275.

49. Naya, S.; Tokunaka, T.; Nitta, M. J. Org. Chem. 2003, 68, 9317.

50. Wei, X.; Du, Z.-Y.; Zheng, X.; Cui, X.-X.; Conney, A.H.; Zhang, K. European J. Med. Chem. 2012, 53, 235.

51. Conard, C.R.; Dolliver, M.A. Org. Synth. Coll. Vol. 1943, 2, 167.

52. http://www.chemspider.com/Chemical-Structure.4527132.html, accessed May 7, 2014.

53. Iranpoor, N.; Kazemi, F. Tetrahedron 1998, 54, 9475.

54. http://www.chemspider.com/Chemical-Structure.4651672.html, accessed May 7, 2014.

55. Zooroh, H.H.; Abou - El Zahab, M.M.; Abdel - Mogib, M.; Ismail, M.A. Tetrahedron 1996, $52,10147$.

56. Kolev, T.; Bakalska, R.; Seidel, R.W.; Mayer-Figge, H.; Oppel, I.M.; Spiteller, M.; Sheldrick, W.S.; Koleva, B.B. Tetrahedron: Asymmetry 2009, 20, 327.

57. Günter, H. NMR Spectroscopy, Basic Principles, Concepts, and Applications in Chemistry, 2ed ed., John Wiley and Sons: Chichester, UK; 1995.

58. Balci, M. Basic ${ }^{1} H$ and ${ }^{13} C$ NMR Spectroscopy, 1st ed., Elsevier: Amsterdam, The Netherlands; 2005.

59. Rigaku/MSC, Inc., 9009 new Trails Drive, The Woodlands, TX 77381, 2002.

60. Sheldrick, G.M., SHELXS97 and SHELXL97, University of Göttingen, Germany, 1997. 